\title{
« PLACER LE CITOYEN AU CAUR DU SYSTÈME » : ORIGINE ET FONDEMENTS D'UNE IDÉE POPULAIRE
}

\section{Cléa Iavarone-Turcotte*}

Le présent article porte sur une idée qui semble aujourd'hui être sur toutes les lèvres. Une idée du reste avancée aussi bien par des observateurs du monde judiciaire (professeurs, journalistes) que par des acteurs qui en sont parties prenantes (avocats, juges, ministres de la Justice) et qui, partant, trouve écho non seulement au sein de la société civile, mais également à l'intérieur du cercle fermé, étanche, que constitue l'appareil judiciaire. Cette idée, c'est celle de placer le citoyen au coeur du système de justice civile.

Dans l'optique de mieux comprendre cette proposition, qu'elle rebaptise "l'idée de recentrage ", l'auteure s'emploie à la contextualiser. Elle mène cet exercice de contextualisation en deux temps. Ainsi, elle se penche d'abord sur les contextes d'émergence de l'idée de recentrage, avant de mettre au jour ses fondements justificatifs ou sa philosophie sous-jacente.

This article looks into an idea which, today, seems to be on everyone's lips. An idea put forward by observers of the legal world (academics, journalists) as well as by actors and decision makers taking part in it, and that is, thus, echoed not only in civil society, but also within the legal community's inner circle. This idea is the following: to put the citizen at the very heart of the civil justice system.

To assist in understanding this idea, which she calls "recentring", the author provides the context in which it emerged. She does so in a two-step process: she first examines the background against which this recentring was first proposed; she then identifies its underlying justification, or the philosophy on which it is based.

« Le discours sur la justice doit changer. Au-delà des élans romantiques sur la sublimité de la justice et sur la pratique du droit comme sacerdoce (la recherche de la Justice avec un grand J), il faut rappeler que l'activité judiciaire est d'abord et avant tout un service public. On évitera alors de définir ses dysfonctionnements et ses archaïsmes comme des vertus et on replacera le justiciable au cœur du système. $~^{1}$

Lawyer and Doctor of Civil Law (DCL) candidate, McGill Faculty of Law.

Jacques Lachapelle et al, « Des solutions pour la justice civile », Le Devoir de Montréal (28 mars 2008), en ligne :

$<$ http://www.ledevoir.com/non-classe/182426/des-solutions-pour-la-justice-civile> [Lachapelle et al, « solutions »] (nous soulignons). 
Ainsi s'expriment les membres de l'Observatoire du droit à la justice ${ }^{2}$ dans un article paru dans Le Devoir en 2008. Ce regroupement de chercheurs et de professionnels du droit énonce, dans cet extrait, l'idée d'un nécessaire recadrage de la justice civile autour du citoyen dans une de ses formulations les plus classiques. Il s'agit de replacer (ou, diront certains, de «placer $»^{3}$ ), le justiciable, le citoyen ${ }^{4}$ ou encore la personne $^{5}$ au cœur du système judiciaire, en son centre ${ }^{6}$. D'autres rendent la même idée en des

2 L'Observatoire est une « entité fondée sur la collaboration de praticiens, de penseurs et de chercheurs préoccupés par le problème de l'accès à la justice » (ibid). Voir « Observatoire du droit à la justice », en ligne, Centre de recherche en droit public : <http://www.crdp.umontreal.ca/recherche/groupes/observatoire-du-droit-a-la-justice/>.

3 Voir par exemple Georges De Leval, « Le rapport Guinchard ou la réponse idoine aux attentes du justiciable belge », dans Justices et droit du procès : du légalisme procédural à l'humanisme processuel : mélanges en l'honneur de Serge Guinchard, Paris, Dalloz, 2010, 133, à la p 144. Notons que le choix du terme « placer» peut sembler plus juste, dans la mesure où le justiciable n'ayant jamais véritablement été, dans les faits, au cœur du système de justice civile, il ne pourra pas y être « replacé » ou « remis », mais bien seulement « placé » ou « mis ».

4 Dans la documentation consultée, on prône un recadrage de la justice civile aussi bien autour de la personne du justiciable que de celle du citoyen, les deux termes étant même parfois employés indistinctement (aux côtés d'autres tels que « usager », « utilisateur », « client», « consommateur », ou encore « public »). Toutes ces appellations renvoient au profane en droit; c'est ce dernier qui est visé par l'idée de recentrage. Nous privilégierons pour notre part le terme " citoyen », pour marquer le fait que le discours de recentrage ne se confine pas au cas de l'usager du système, donc au justiciable, mais s'étend également au membre de la société civile, donc au citoyen. Si l'on retient « citoyen », c'est aussi parce que ce terme, plus large, « contient » le justiciable éventuel : «l'usager se cache dans tout citoyen qui, un jour ou l'autre peut avoir recours à l'institution judiciaire » (Simone Gaboriau, « Le rôle des usagers dans le fonctionnement du système judiciaire. Les moyens d'associer les usagers à la gestion des tribunaux », en ligne, Conseil de l'Europe : $<$ http://www.coe.int/t/dghl/cooperation/cepej/thematiques/Citoyens/gaboriauF.pdf $>$ [Gaboriau]. Voir également Pierre Bauby, « De l'usager au consommateur : le citoyen, moteur de la qualité », dans Hélène Pauliat, dir, La qualité, une exigence pour l'action publique en Europe?, Limoges, Pulim, 2004, 101, à la p 104 [Bauby] (où 1'auteur exprime l'opinion que chaque individu est à la fois consommateur, usager et citoyen).

5 Le Comité de l'accès à la justice de l'Association du Barreau canadien justifie ainsi sa préférence pour le terme « personne » : « Nous appelons souvent les personnes qui ont affaire au système de justice des “ clients" ou des " utilisateurs ", mais le Comité a choisi plutôt d'utiliser autant que possible " personnes " pour éviter de réduire la place de chacun dans le système de justice à une catégorie passive de récipiendaires de services ». Voir Association du Barreau canadien, Justice pour tous - trouver l'équilibre. Rapport Atteindre l'égalité devant la justice : une invitation à l'imagination et à l'action, Ottawa, Association du Barreau canadien, 2013, à la p 10 [Association du Barreau canadien, Justice pour tous].

6 On retrouve une formulation identique ou très similaire dans les sources suivantes : Michel Bouchard, « Une réforme nécessaire ", (1999) 40:1 C de D 7, à la p 8 [Bouchard]; Comité de révision de la procédure civile, La révision de la procédure civile - Une nouvelle culture judiciaire, Québec, ministère de la Justice, 2001, à la p 34 [Comité de révision de la procédure civile]; Lise I Beaudoin, « Réforme de la procédure civile - une nouvelle culture judiciaire s'impose à tous », Le Journal - Barreau du Québec (octobre 2001), en ligne : <http://www.barreau.qc.ca/pdf/journal/vol33/no16/cpc.html> [Beaudoin]; Commission sur la répartition des contentieux, L'ambition raisonnée d'une justice apaisée - rapport au Garde des Sceaux, Paris, La documentation française, 2008, notamment aux pp 39, 40, 43 et 181 [Commission sur la répartition des contentieux]; Pierre Noreau, « Avenir de l'institution judiciaire et difficultés du changement institutionnel : attentes des citoyens et nécessités de la justice », dans Tribunaux de première instance : vision d'avenir - Actes du colloque 2004, Montréal; Québec, Conseil de la magistrature du Québec, 2005, 41, à la p 52 [Noreau, «Avenir de 1'institution judiciaire »]; Hélène Buzzetti, « La justice sur la voie d'accès », Le Devoir de Montréal (23 décembre 2014), en ligne : <http://www.ledevoir.com/societe/jus-tice/427436/la-justice-sur-la-voie-dacces > [Buzzetti]; Pierre Noreau, « La justice est-elle soluble dans la procédure ? Repères sociologiques pour une réforme de la justice civile », (1999) $40 \mathrm{C}$ de D 33, à la p 48 [Noreau, « justice »]. 
termes quelque peu différents, prônant l'avènement d'une justice civile qui soit « axée sur le citoyen »" c'est-à-dire véritablement conçue pour celui-ci. Les différentes formulations de ce que nous appellerons « l'idée de recentrage » abondent, et il serait vain (et impossible) de rapporter chacune d'entre elles. Il nous semble davantage intéressant de souligner que leur lecture successive révèle l'existence d'un certain consensus chez leurs auteurs, consensus qui tient à ce que cette idée implique lorsqu'elle est prise dans sa plus simple expression. Placer le citoyen au centre du système de justice civile, ce serait, au minimum, prêter l'attention qu'il se doit à ses attentes et/ou à ses besoins, et voir à y répondre ${ }^{8}$. Autrement dit, le recentrage irait de pair avec la satisfaction des attentes et des besoins des citoyens en matière de justice civile 9 .

Pourquoi nous pencher sur l'idée de recentrage ? Essentiellement en raison de la prégnance actuelle de deux phénomènes dont aucun juriste ne peut aujourd'hui nier l'importance et la gravité. Le premier, dont il sera question ci-après, est la crise de l'accès à la justice civile. L'ampleur de cette crise et l'urgence d'y faire face commandent, à notre sens, que l'on porte notre regard sur le citoyen, afin de repenser la justice en fonction de celui-ci et non plus des seuls acteurs du milieu judiciaire. Lié au problème d'inaccessibilité économique de la justice sans pour autant qu'il ne s'explique que par lui, le second phénomène est celui de l'autoreprésentation. Les justiciables sont en effet de plus en plus nombreux à se présenter seuls en cour ${ }^{10}$, une tendance qui ne semble pas vouloir s'inverser. À notre sens, autant que l'enjeu plus large de l'accès à la justice civile, celui que représente l'autoreprésentation

7 Voir par exemple Comité de réflexion et d'orientation sur la justice de première instance au Québec, Une réforme judiciaire axée sur le citoyen, avril 2005, aux pp 4 et 22, en ligne, Cour du Québec :

$<$ http://www.tribunaux.qc.ca/c-quebec/CommuniquesDocumentation/reformeJudiciaireCitoyen.pdf> [Comité de réflexion et d'orientation sur la justice de première instance au Québec]. Voir également Comité d'action sur l'accès à la justice en matière civile et familiale, L'accès à la justice en matière civile et familiale - une feuille de route pour le changement, Ottawa, Comité d'action sur l'accès à la justice en matière civile et familiale, octobre 2013, aux pp i et 8 [Comité d'action sur l'accès à la justice en matière civile et familiale, feuille de route] et Association du Barreau canadien, Justice pour tous, supra note 5 aux pp 14-15, 66 et 104.

8 Tous les tenants d'un recadrage de la justice civile autour de la personne du citoyen ne s'entendent pas forcément sur ce que ce recentrage signifie précisément ou implique concrètement. Autrement dit, le sens exact de cette idée varie en fonction de celui qui la formule ou l'endosse. Certains se montrent, à cet égard, plus conservateurs et d'autres, plus ambitieux. Cela dit, malgré une absence de consensus quant au sens précis à donner à l'idée de recentrage, tous (ou presque) l'accolent à celle d'une nécessaire prise en considération des attentes et des besoins des citoyens en matière de justice civile. Si nous écrivons ici « au minimum », c'est donc pour marquer le fait que cette prise en considération des attentes et des besoins des citoyens constitue, en quelque sorte, le « sens minimal » de l'idée de recentrage, le «plus petit dénominateur commun » des textes qui la mettent de l'avant.

9 Voir entre autres Canadian Bar Association, Systems of Civil Justice Task Force Report, Ottawa, Canadian Bar Association, 1996, aux pp 3, 7, 23, 28, 55 et 56 [Canadian Bar Association, Task Force Report]; Bouchard, supra note 6 aux pp 7 et 9; Noreau, «Avenir de l'institution judiciaire», supra note 6 à la p 51; Association du Barreau canadien, Justice pour tous, supra note 5 aux pp 65-66; Pierre-Claude Lafond, L'accès à la justice civile au Québec-portrait général, Cowansville, Yvon Blais, 2012, à la p 311 [Lafond, L'accès à la justice civile]; Lachapelle et al, « solutions », supra note 1 et British Columbia Ministry of Justice, White Paper on Justice Reform part two: A Timely, Balanced Justice System, British Columbia Ministry of Justice, février 2013, aux pp 2, 3, 4, 7 et 11 [British Columbia Ministry of Justice, White Paper].

10 Ce qui, on le sait, n'est pas sans conséquences, autant pour les parties non représentées que pour les avocats, les juges et le personnel judiciaire. Plus fondamentalement, c'est tout le fonctionnement du système accusatoire, basé sur le principe de la représentation des parties, qui est chamboulé lorsqu'une des parties n'a pas d'avocat. 
change fondamentalement la donne, et justifie que l'on réfléchisse à la justice en prenant comme point de départ, ce faisant, la personne du citoyen.

Quant à l' " utilité scientifique » de notre article - notre contribution à l'avancement des connaissances, pour reprendre la formule bien connue -, elle découle notamment du fait que nous abordons ici un sujet d'actualité, et ce, d'une manière que nous souhaitons originale et novatrice. En effet, à en juger par la recherche que nous avons effectuée, il appert que personne ne s'est encore intéressé à l'idée de recentrage comme nous le faisons, c'est-à-dire en s'y attardant pour elle-même, indépendamment du contexte dans lequel elle prend racine. C'est-à-dire que, au regard des sources consultées, cette idée s'inscrit plus souvent qu'autrement dans un argumentaire plus large qui la dépasse. Autrement dit, elle ne constitue que très rarement le sujet précis retenu par l'auteur; elle est, plus souvent, soulevée par celui-ci à l'occasion d'une démonstration ou d'un propos qui est autre. Partant, nous nous distinguons de cette documentation existante en considérant directement l'idée de recadrage de la justice civile autour du citoyen. Notre contribution tient également à notre approche "intégrée ». Nous avons en effet cherché à transcender les frontières propres aux différents domaines du droit dans lesquels l'idée de recentrage est avancée, pour nous permettre de rassembler, dans un même article, une information actuellement dispersée.

$\mathrm{Au}$ regard de la revue de littérature effectuée, la récurrence de l'idée de concevoir une justice civile orientée vers l'usager est telle qu'il est permis de parler de l'existence d'un véritable discours la portant. Or si ce discours peut sembler, d'emblée, quelque peu utopique (surtout à des juristes), il mérite toutefois que l'on s'y arrête. L'intérêt qu'il présente à nos yeux ne tient pas seulement au nombre des personnes qui y adhérent. Il découle également du fait que ces personnes sont d'horizons divers, en ce qu'elles comptent autant des observateurs du monde judiciaire (par exemple, des professeurs de droit) que des acteurs qui en sont partie prenante (des avocats, des juges, voire même des ministres de la Justice, on le verra). C'est donc dire que le plaidoyer en faveur d'une justice civile centrée sur le justiciable émane non seulement de l'extérieur, mais également de l'intérieur même du système judiciaire. Partant, à notre avis, le fait que des personnes aux intérêts aussi différents (parfois même divergents) viennent suggérer, au final, la même idée, fait en sorte de crédibiliser celle-ci. Ajoutons que les tenants de cette idée sont issus de juridictions diverses, ce qui lui confère un caractère international et accroît, à nouveau, l'intérêt que nous lui portons.

Cela dit, l'objectif du présent article demeure modeste. Il s'agira essentiellement de mieux comprendre l'idée de concevoir une justice civile tout entière tournée vers le citoyen en la contextualisant. Cet exercice de contextualisation sera mené en deux temps. D'abord, nous nous emploierons à situer l'idée de recentrage dans le temps et dans l'espace, afin de mettre au jour son, ou plus justement ses, contextes d'émergence (I). Nous nous pencherons ensuite sur les fondements justificatifs de cette idée, sur sa philosophie sous-jacente, s'il en est une (II).

\section{LES CONTEXTES D'ÉMERGENCE DE L'IDÉE DE RECENTRAGE}

L'idée de concevoir le système de justice civile en prenant comme point de départ la personne du citoyen est-elle ancienne ou nouvelle? Plusieurs auteurs y voient une tendance contemporaine ${ }^{11}$,

11 Nous disons bien ici contemporaine et non récente, parce qu'en matière de réforme de la justice civile, un mouvement vers une cour « orientée vers l'usager » serait perceptible depuis le début des années 1990, du moins en Amérique du 
laquelle aurait émergé dans trois contextes connexes, mais non pas moins distincts : l'accès à la justice civile (A), la réforme de cette justice (B) et, enfin, l'évaluation de celle-ci (C). Nous examinerons successivement ces trois contextes d'émergence.

\section{A. L'accès à la justice civile}

L'idée de recentrage est tout d'abord défendue dans des sources récentes plaidant, plus largement, pour un meilleur accès à la justice en matière civile ${ }^{12}$. Par exemple, dans son rapport final rendu public en octobre 2013, le Comité d'action sur l'accès à la justice en matière civile et familiale ${ }^{13}$ fait de l'idée de « donner la priorité au public » l'un des six principes directeurs à la base de l'important changement de culture qu'il prône, et qui lui parait inévitable pour que se concrétise enfin l'avènement d'un meilleur accès à la justice au Canada ${ }^{14}$. Il s'agit, pour le Comité, de «modifier la cible principale de nos efforts », en cessant de diriger ceux-ci vers l'intérieur du système, et en les axant plutôt « sur les personnes qui utilisent [ce] système $»^{15}$. Le Comité rejoint à ce propos l'Association du Barreau canadien $(\mathrm{ABC})$ qui,

Nord et en Australie. Voir à ce propos Steven Parker, Courts and the Public, Carlton South, Australian Institute of Judicial Administration Incorporated, 1998, aux pp 1 (où le professeur fait état, pour ce qui est de l'Australie, d'un « movement since the early 1990s towards a greater 'consumer-orientation' in the courts ») et 23-24 (où il écrit, en 1998, ce qui suit : "The movement towards a more consumer-oriented court began some years ago in Australia, and earlier in North America. In 1990, the need for the courts to become more "user-friendly" was highlighted by Professor Thomas Church in his hard-hitting AIJA Annual Oration in Judicial Administration, although the idea had been gestating for some time. (...) By 1993, the then Executive Director of the AIJA, Professor Peter Sallmann, was able to write that it was "very clear" that a "consumer orientation" had begun to appear in Australian courts. » (ibid, références omises; nous soulignons). Le professeur nuance ensuite son propos en soulignant que bien que ce mouvement des cours australiennes vers une « orientation usager » soit bel et bien amorcé, le chemin à parcourir à ce chapitre demeure important (ibid à la p 29) [Parker].

12 C'est notamment le cas des sources suivantes : Comité d'action sur l'accès à la justice en matière civile et familiale, feuille de route, supra note 7; Comité d'action sur l'accès à la justice en matière civile et familiale, Compte rendu du Colloque, Ottawa, Comité d'action sur l'accès à la justice civile et familiale, juin 2014 [Comité d'action sur l'accès à la justice en matière civile et familiale, Compte rendu du Colloque]; Association du Barreau canadien, Justice pour tous, supra note 5; Diana J Lowe, «Access to Justice: Responding to Public Needs Within a Culture of Proportionality », dans Pierre Noreau, dir, Révolutionner la justice - Constats, mutations et perspectives, Les journées Maximilien-Caron 2009, Montréal, Thémis, 2010, 84 [Lowe]; Action committee on access to justice in civil and family matters, Prevention, triage and referral working group, Final Report - Responding Early, Responding Well: Access to Justice through the Early Resolution Services Sector, 12 février 2013 [Action committee on access to justice in civil and family matters, Prevention, triage and referral working group] et Maurits Barendrecht et al, Towards Basic Justice Care for Everyone: Challenges and Promising Approaches. Trend Report I Part 1, Hague Institute for the Internationalization of Law (HiiL), 2012 [Barendrecht et al].

13 Créé en 2008 par la juge en chef de la Cour suprême du Canada, l'honorable Beverley McLachlin, et présidé par le juge Thomas A. Cromwell, le Comité est formé de chefs de file du milieu de la justice civile et familiale ainsi que d'un représentant du public. Voir Comité d'action sur l'accès à la justice en matière civile et familiale, Compte rendu du Colloque, supra note 12 à la p 1.

14 Comité d'action sur l'accès à la justice en matière civile et familiale, feuille de route, supra note 7 aux pp iv et 8 . Voir également les pages 2,12 et 28 .

15 Ibid à la $\mathrm{p} 8$. Voir dans le même sens le Groupe de travail sur la prévention, le triage et l'aiguillage du Comité d'action sur l'accès à la justice en matière civile et familiale : « This report begins by establishing a vision of access to justice that focuses first and foremost on the needs and concerns of individuals » (Action committee on access to justice in civil and family matters, Prevention, triage and referral working group, supra note 12 à la p i; voir également la page 35). 
elle aussi, plaide pour la mise en place d'un système de justice civile qui soit véritablement « axé sur les personnes ${ }^{16}$. De l'avis de l'ABC, il s'agit essentiellement de "mettre l'accent sur les besoins des utilisateurs et la façon dont ils ont besoin qu'ils soient comblés ${ }^{17}{ }^{17}$. À cette fin, l'ABC propose une nouvelle vision du système judiciaire articulée autour de six engagements concrets, dont le premier est de chercher à répondre en priorité aux besoins des personnes plutôt qu'à ceux des juristes :

«Un système de justice inclusif doit être également accessible à tous, quels que soient leurs moyens, leurs capacités ou leur situation sociale. Il exige six engagements concrets :

1. Les personnes - Le système met l'accent sur les besoins des personnes, et non ceux des professionnels de la justice et des institutions. (...) ${ }^{18}$

L'attention toute particulière portée aux besoins des citoyens, parfois désignée comme l'approche «centrée sur le client ou l'usager " ${ }^{19}$, correspondrait à l'évolution la plus récente du mouvement vers une justice plus accessible ${ }^{20}$, mouvement amorcé aux États-Unis dans les années 1960 et ayant connu, depuis, plusieurs vagues successives ${ }^{21}$. Cette orientation nouvelle des efforts en matière d'accessibilité à la justice a été marquée par le développement des connaissances en matière de besoins juridiques (« legal needs »), besoins qui, lorsqu'ils ne sont pas comblés, ont des répercussions importantes sur la vie des gens comme sur la société dans son ensemble ${ }^{22}$. Les débuts de la recherche empirique sur ces besoins ainsi que sur les problèmes juridiques tels qu'ils sont réellement vécus par ceux qui les rencontrent remontent à 1999, avec la publication d'une étude phare sur le sujet émanant du Royaume$\mathrm{Uni}^{23}$. Depuis, dans la foulée de ce projet novateur, plus de vingt études empiriques portant sur les besoins juridiques en matière civile ont été menées, et ce, dans pas moins de treize États ${ }^{24}$, dont le Canada $^{25}$. C'est donc dire que, en matière d'accès à la justice, l'approche axée sur les besoins juridiques des individus serait non seulement récente, mais correspondrait au surplus à une tendance internationale. Notons à ce propos que, dans son rapport de février 2013, le Groupe de travail sur la prévention, le triage et l'aiguillage du Comité d'action sur l'accès à la justice en matière civile et familiale, prenant

16 Association du Barreau canadien, Justice pour tous, supra note 5 aux pp 14-15. Voir aussi les pages 66 et 104.

$17 \quad$ Ibid à la p 66.

18 Ibid à la p 65.

19 Cette appellation est plus courante dans la documentation en langue anglaise, où l'on parle d'une « client-centered or user-focused approach ». Voir par exemple Lowe, supra note 12 à la p 86.

20 Voir entre autres Association du Barreau canadien, Justice pour tous, supra note 5 à la p 64. Sur l'idée de nouveauté en matière d'accès à la justice, voir également Barendrecht et al, supra note 12 à la p 3 .

21 Pour un résumé de ces vagues successives et un compte-rendu de diverses interprétations du mouvement d'accès à la justice dans le temps, voir Lafond, L'accès à la justice civile, supra note 9 aux pp 20 à 23.

22 Association du Barreau canadien, Justice pour tous, supra note 5 à la p 64.

23 Nous en référons à Hazel Genn et Alan Paterson, Paths to Justice: What people do and think about going to law, Scotland, Hart Publishing, 1999.

24 Michael Trebilcock, Anthony Duggan et Lorne Sossin, « Introduction », dans Michael Trebilcock, Anthony Duggan et Lorne Sossin, dir, Middle Income Access to Justice, Toronto, University of Toronto Press, 2012, 3, à la p 7. Au sujet de ces études (et pour un aperçu de ce que la somme de celles-ci révèle), voir également Action committee on access to justice in civil and family matters, Prevention, triage and referral working group, supra note 12 à la p 4.

25 Pour un aperçu d'un projet actuellement mené au Canada en la matière, voir « Everyday Legal Problems and The Cost of Justice in Canada », en ligne, Forum canadien sur la justice civile : <http://www.cfcj-fcjc.org/cost-of-justice $>$. 
comme point de départ les problèmes juridiques pour traiter d'accès à la justice, s'autorise justement de cette tendance internationale :

« [The] primary starting point and consistent focus [of this report] is on the broader range of problems experienced by the public, not just those that are adjudicated in courts. It looks at legal problems from the point of view of the people experiencing them. This approach is in keeping with current innovative trends and thinking internationally $(\ldots) »^{26}$

À l'appui de l'idée d'« internationalité » de l'approche fondée sur les besoins juridiques, le Groupe de travail cite le Hague Institute for the Internationalization of Law (HiiL), dans un passage qu'il convient de reproduire ci-après :

« More recently researchers and reports have begun to focus on new approaches, more bottom up, working from the problems people actually experience and from their capabilities. (...) We will call these initiatives justice needs approaches, because they have in common that they start from the actual problems that people experience and their capabilities to solve them. These capabilities are then strengthened. This can be done by local initiatives (...). But (...) justice needs approaches can also be scaled up across borders, and stimulated by new types of codification, precisely because they start from people's problems and not from the laws or the legal system that has been built in their country. $»^{27}$

Le professeur Jean-François Roberge note lui aussi l'existence d'une « international trend toward taking into account the public's experiences with and perceptions of justice $»^{28}$. Pour lui, le choix fait par le Groupe de travail d'ancrer sa réflexion dans la notion de besoins juridiques révèle l'émergence, au Canada, d'une approche nouvelle en matière d'accès à la justice : l'approche contextuelle ${ }^{29}$. Cette approche, qui gagnerait du terrain face à l'approche institutionnelle ayant traditionnellement prévalu au pays $^{30}$, prend comme point de départ les attentes de justice. Il écrit: "This vision focuses on the expectations of justice and the ability of litigants to achieve them in order to enhance the sense of justice felt during and after use of the judicial system $»^{31}$. L'objectif est alors d'adapter le système judiciaire afin de faire en sorte de rencontrer les attentes citoyennes ${ }^{32}$.

26 Action committee on access to justice in civil and family matters, Prevention, triage and referral working group, supra note 12 à la $\mathrm{p} 3$ (nous soulignons).

27 Barendrecht et al, supra note 12 à la p 15 (nous soulignons).

28 Jean-François Roberge, « Perspectives on Access to Justice and Dispute Prevention and Resolution: The Canadian Experience », (2013) 17 Dutch-Flemish Mediation \& Conflict Management J 2, 17-18 [Roberge, «perspectives »].

29 Ibid à la p 17. Voir également les pages 14,16 et 18.

30 Et qui y serait encore dominante, en particulier au Québec, malgré les avancées récentes de l'approche contextuelle; voir ibid aux pp 14-15. Au sujet de l'approche institutionnelle, voir ibid aux pp 14-16.

31 Ibid à la $\mathrm{p} 13$. Pour de plus amples éléments de réflexion sur l'approche contextuelle de l'accès à la justice, voir JeanFrançois Roberge, La justice participative - Changer le milieu juridique par une culture intégrative de règlement des différends, Cowansville, Yvon Blais, 2011, aux pp 27 à 32 [Roberge, La justice participative].

32 Roberge, «perspectives », supra note 28 à la p 14. 
L'accent mis sur la personne du citoyen ou du justiciable dans les développements récents en matière d'accès à la justice ressort également de plusieurs initiatives de mesure de l'accès à la justice ${ }^{33}$, certaines d'entre elles prenant appui sur le corpus croissant de connaissances en matière de besoins juridiques. Par exemple, le Tilburg Institute for Interdisciplinary Studies of Civil Law and Conflict Resolution Systems [TISCO] met de l'avant une méthodologie de mesure des coûts et de la qualité de l'accès à la justice qui prend comme point de départ la perspective de l'usager ${ }^{34}$ (entendu ici comme la partie «active », cherchant à obtenir justice et engageant une procédure en ce sens, que cette dernière soit judiciaire ou extrajudiciaire, complexe ou simple ${ }^{35}$ ). Le caractère distinctif de cette approche méthodologique réside dans l'angle « subjectif » retenu. Elle postule en effet qu'une mesure véritable de l'accès à la justice devra nécessairement prendre en considération la perspective des utilisateurs, ce qui implique de recueillir leurs points de vue quant à l'expérience judiciaire qu'ils ont vécue. Ces perceptions des justiciables, nécessairement subjectives, sont posées comme indicateurs valides permettant d'évaluer la justice, au même titre (et parfois aux côtés) d'indicateurs «objectifs » plus traditionnels, et souvent quantitatifs (p. ex., « inputs », « outputs », opinions d'experts, etc.). À fortiori, en vertu de cette méthodologie, l'usager, justement parce qu'il a fait l'expérience concrète d'une procédure menant (ou non) à la justice (ce que l'on appelle le « path to justice »), constitue la personne la plus à même de juger des coûts et de la qualité de cette dernière. C'est pourquoi il convient de mesurer l'accès à la justice en partant d'abord de son expérience et de ses perceptions ${ }^{36}$. Notons au passage la similitude de cette vision avec celle du professeur Roberge, qui choisit, pour sa part, non pas de mesurer, mais plutôt de définir l'accès à la justice en en référant aux perceptions, motivations et attentes du citoyen :

« L'accès à la justice est l'action de rendre accessible (1) une forme de justice (2) qui doit être perçue comme une forme de justice par celui ou celle à qui elle est destinée et (3) qui répond à ses motivations et attentes de justice. $\gg{ }^{37}$

Au regard de cette définition, l'un des défis à relever en matière d'accès à la justice devient alors, de l'avis du professeur, l'adéquation - ou, à défaut de cette dernière, le rapprochement - de l'offre de justice, d'une part, et de la perception, des besoins et des attentes des citoyens en matière de justice, d'autre part $^{38}$.

33 Pour un aperçu de cinq de ces initiatives, voir Canadian Bar Association, Access to Justice Metrics - A Discussion Paper. Envisioning Equal Justice, An Initiative of the Canadian Bar Association, avril 2013 [Canadian Bar Association, Access to Justice Metrics].

34 Tilburg Institute for Interdisciplinary Studies of Civil Law and Conflict Resolution Systems / TISCO, Martin Gramatikov et al, A Handbook for Measuring the Costs and Quality of Access to Justice, Apeldoorn; Antwerpen; Portland, Maklu \& Tilburg Institute for Interdisciplinary Studies of Civil Law and Conflict Resolution Systems, 2009, à la p 9.

35 Ibid aux pp 24 et 28.

36 Ibid à la p 27.

37 Roberge, La justice participative, supra note 31 à la p 23 (nous soulignons).

38 Ibid aux pp 23-24. Le professeur Roderick A. MacDonald plaide aussi pour l'avènement d'une « citizen-centered conception of access to justice » (voir Roderick A MacDonald, « Access to Civil Justice », dans Peter Cane et Herbert M Kritzer, dir, The Oxford handbook of empirical legal research, Oxford; New York, Oxford University Press, 2010, 492, à la p 495. Voir aussi les pages 513, 516 et 518). 


\section{B. La réforme de la justice civile}

Si l'idée de recentrage est particulièrement patente au regard des développements récents en matière d'accessibilité à la justice dont nous venons de faire état, elle ne se confond pas pour autant avec l'objectif d'un meilleur accès à la justice. Il nous paraît important de le souligner puisque, intuitivement, on pourrait penser que l'idée de placer le citoyen au cœur du système judiciaire se résume essentiellement au fait de lui faciliter l'accès à un tel système. Autrement dit, plaider pour un recentrage reviendrait à plaider pour un accès accru à la justice. Or la question d'un possible recadrage de la justice autour du citoyen n'est pas tout entière contenue dans celle de l'accès à la justice. On en veut pour preuve le fait que cette idée a également émergé, est également défendue, dans d'autres contextes, dont celui de la réforme de la justice civile ${ }^{39}$, dont il sera maintenant question.

Précisons d'emblée que nous parlons de "réforme de la justice civile » au sens large, pour y faire entrer, d'abord, la révision de la procédure civile, mais également la réforme de l'organisation ou de la structure judiciaire et, enfin, la modernisation des systèmes de justice civile plus généralement. Nous examinerons successivement ces trois cas de figure.

\section{Révision de la procédure civile}

En premier lieu, au regard de la documentation consultée, l'idée de mettre le citoyen au centre du système de justice civile est avancée dans le contexte d'une révision de la procédure civile ${ }^{40}$. Pour s'en convaincre, il suffit de se rapporter au cas de la réforme du Code de procédure civile du Québec. Déjà, en 1945, la Loi pour améliorer le Code de procédure civile ${ }^{41}$ pose, parmi ses objectifs, celui de faire en sorte que la procédure « soit mieux adaptée aux besoins »" ${ }^{42}$. En 1975, le ministre de la Justice Jérôme Choquette, en avant-propos de son désormais célèbre Livre blanc intitulé La justice contemporaine ${ }^{43}$, écrit que « L'indispensable autorité de la justice dans toute société démocratique doit reposer sur des prémisses d'intégrité et d'impartialité, comme sur des institutions et des lois adaptées à leur époque et

39 Cela est d'autant plus vrai que l'idée de recentrage a été soulevée dans le contexte d'une réforme de la justice civile bien avant que les auteurs traitant d'accès à la justice ne s'y intéressent. Rappelons en effet que, en matière de réforme, l'idée de placer le citoyen au cœur du système remonterait au début des années 1990 (voir supra à la n 11). Aujourd'hui, l'approche centrée sur l'usager ou le client serait commune en ce domaine (voir notamment Kari D Boyle, « What Does a "user-centred” Approach Really Mean? » (13 mars 2015), Slaw, en ligne : <http://www.slaw.ca/20-15/03/13/whatdoes-a-user-centred-approach-really-mean/> [Boyle]).

40 Voir en guise d'exemples Noreau, « justice », supra note 6 aux pp 37 et 48 (« Il est tout à fait opportun donc de réfléchir à la révision des mécanismes qui balisent la procédure civile, mais sans doute doit-on le faire dans une perspective qui replace le justiciable au centre du travail des tribunaux, de manière que cette réforme de la procédure civile soit également une réforme de la « justice » civile (...)»- nous soulignons); Alan Uzelac, « Turning civil procedure upside down: from judges' law to users' law », dans Tweehonderd jaar/Bicentenaire Code de Procédure civile, Mechelen, Kluwer uitgevers, 2008, 413, à la p 425 [Uzelac, « upside down »] (où l'auteur exprime l'avis qu'une « orientation towards users » constituera l'une des caractéristiques principales de la procédure civile du futur), et Teresa Arruda Alvim Wambier, «Judicial Activism as Goals Setting - Civil Justice in Brazil », dans Alan Uzelac, dir, Goals of civil justice and civil procedure in contemporary judicial systems, Londres, Springer, 2014, 245, à la p 257.

$41 \quad$ (1945) 9 Geo VI, c 69.

42 Tel qu'il est rapporté dans Marie José Longtin, «Les perspectives de révision du Code de procédure civile », dans Nicholas Kasirer et Pierre Noreau, dir, Sources et instruments de justice en droit privé, Montréal, Thémis, 2002, 195, à la p 198.

43 Jérôme Choquette, La justice contemporaine, Québec, ministère de la Justice, 1975, avant-propos [Choquette]. 
aux besoins des citoyens $"{ }^{44}$. Puis, en 1999, c'est au tour du sous-ministre de la Justice Michel Bouchard d'affirmer, en ce qui a trait à l'exercice de révision du Code de procédure civile entrepris au printemps 1998, qu'il « devrait se traduire par une justice civile qui prendra mieux en considération les attentes et les besoins des citoyens, des justiciables (...) » ${ }^{45}$. Il tient alors les propos suivants :

«Principal intéressé par l'administration de la justice, le citoyen est trop souvent le grand laissé-pour-compte de nos réflexions et de nos décisions. Pourtant, comment pourrionsnous justifier toute notre organisation de la justice si aucun citoyen ne faisait appel à ces services? Le citoyen doit être au cœur de l'administration de la justice. Et il doit pouvoir jouer un rôle dans la réforme de la procédure civile. $\gg{ }^{46}$

Toujours dans le contexte de la révision du Code de procédure civile amorcée en 1998, notons que la nécessité de tenir compte des attentes et des besoins des justiciables fait expressément partie du mandat confié au Comité de révision de la procédure civile par le ministre de la Justice de l'époque, M. Serge Ménard $^{47}$. Dans son rapport déposé en juin 2001, le Comité se dit ainsi «(...) préoccupé de répondre aux attentes des justiciables qui, au premier chef, auront recours au code pour faire valoir leurs droits $»^{48}$. Ce faisant, il importe, toujours selon le Comité, « (...) d'amener le justiciable à prendre conscience de la place primordiale qui lui revient dans le système judiciaire (...) ${ }^{49}$. Notons que ces derniers extraits sont tirés de la section du rapport dans laquelle le Comité met de l'avant sa vision de la procédure civile. L'idée de recentrage ressort également des objectifs de la révision de la procédure civile tels qu'ils sont énoncés dans le rapport Ferland. La section du rapport consacrée à l'énoncé de ces objectifs contient en effet l'affirmation suivante : «Le justiciable doit être placé au cœur du système de justice $\gg{ }^{50}$. Enfin, commentant le dépôt du rapport Ferland, le ministre de la Justice en place en 2001, M. Paul Bégin, exprimera à son tour l'avis suivant : « Le citoyen doit être au cœur du système de justice » 51 .

Pour clore notre propos sur les réformes successives de la procédure civile québécoise, il importe de mentionner que, selon certains, la mise du citoyen au centre du système de justice civile sous-tend également la toute dernière révision du Code de procédure civile. Rappelons en effet que, le 20 février 2014, l'Assemblée nationale adoptait à l'unanimité le projet de Loi instituant le nouveau Code de procédure civile ${ }^{52}$, dotant ainsi le Québec d'un nouveau Code de procédure civile dont l'entrée en

44 Ibid; nous soulignons.

45 Bouchard, supra note 6 à la p 7 (nous soulignons).

46 Ibid aux pp 8-9 (nous soulignons). Dans le même article, il affirme également qu' « (...) il devient primordial de prendre le pouls du citoyen, de bien saisir ses attentes et de mesurer ses besoins. » (ibid à la p 9). Sur les citoyens comme «principaux intéressés » au bon fonctionnement des tribunaux, voir également Matthieu Boissavy et Thomas Clay, Reconstruire la justice, Paris, Odile Jacob, 2006, à la p 28 [Boissavy et Clay].

47 Voir Comité de révision de la procédure civile, supra note 6 à la p 2. Voir également la p 3 : « [Le Comité] doit cependant avoir, comme cela lui fut demandé par le Ministre, le souci de placer le citoyen au cœur de la révision. » (nous soulignons).

48 Ibid à la p 32. Quant à la nécessité de répondre aux besoins des justiciables en sus de leurs attentes, voir la page 36.

49 Ibid à la p 32.

$50 \quad$ Ibid à la p 34. Voir, dans le même sens, la page 3.

51 Propos rapportés dans Beaudoin, supra note 6.

52 PL 28, Loi instituant le nouveau Code de procédure civile, $1^{\mathrm{re}}$ sess, 40 ${ }^{\mathrm{e}}$ lég, Québec, 2013. 
vigueur est prévue pour l'automne $2015^{53}$. Or pour l'Observatoire du droit à la justice, cette réforme «place le justiciable au cœur du système $(\ldots) »^{54}$. Il écrit :

« Cette réforme, souhaitée depuis longtemps par le ministère de la Justice, attendue des milieux universitaire et judiciaire, mérite une vaste opération de communication. Il faut faire connaître au grand public et aux justiciables ses orientations et ses valeurs. Car les justiciables devront apprendre qu'ils sont dorénavant parties prenantes de ce nouveau système de justice et qu'ils en seront les premiers bénéficiaires, ce qui les autorise à entretenir de nouvelles attentes vis-à-vis des praticiens et du système judiciaire dans son ensemble. $\|^{55}$

Précisons que l'Observatoire tempère ensuite ses propos en soulignant que cette révision, bien qu'elle "vise directement le justiciable », échouera néanmoins si elle ne s'accompagne pas d'une " action décisive des acteurs du système judiciaire doublée d'une ferme volonté de changement $»^{56}$.

\section{Réforme de l'organisation judiciaire}

En second lieu, l'idée de recentrage est également présentée dans des textes et rapports portant sur la possible réorganisation du système de justice civile. Par exemple, en 2004, le Conseil de la magistrature du Québec tenait son colloque annuel sur le thème: "Tribunaux de première instance: vision d'avenir », lors duquel furent abordées la question de l'organisation du système judiciaire québécois et celle de l'unification possible des tribunaux judiciaires. Lors de ce colloque, trois conférenciers ont évoqué, dans leur présentation, la nécessité de mener la réflexion en cette matière en veillant bien, ce faisant, à prendre comme point de départ le service au citoyen ${ }^{57}$. L'année suivante, en 2005, le Comité de réflexion et d'orientation sur la justice de première instance au Québec rendait public un rapport intitulé « Une réforme judiciaire axée sur le citoyen $~_{58}$. Dans celui-ci, le Comité se penche sur le bienfondé de la structure judiciaire alors en place en matière de justice de première instance au Québec. Ce faisant, il se dit guidé par la question de savoir si cette organisation judiciaire « répond adéquatement

53 Voir «Le Code de procédure civile», en ligne, Justice Québec : <http://www.justice.gouv.qc.ca/francais/sujets/glossaire/code-proc.htm>.

54 Oscar D'amour et al, « Nouveau Code de procédure civile - Une réforme qui échouera s'il y a indifférence », Le Devoir de Montréal (16 avril 2014), en ligne : <http://www.ledevoir.com/societe/justice/405631/nouveau-code-de-proc>

[D'amour et al, « Nouveau Code de procédure civile »]. Voir dans le même sens Observatoire du droit à la justice, Mémoire de l'Observatoire du droit à la justice présenté à la Commission des institutions de l'Assemblée nationale du Québec dans le cadre de la consultation générale et des auditions publiques sur l'Avant-projet de loi instituant le nouveau Code de procédure civile, décembre 2011, à la p 9 [Observatoire du droit à la justice]. Voir également l'avis en ce sens de Roberge, «perspectives », supra note 28 à la p 18.

55 D'amour et al, « Nouveau Code de procédure civile », supra note 54 (nous soulignons).

56 Ibid.

57 Nous en référons à Noreau, «Avenir de l'institution judiciaire », supra note 6 entre autres aux pp 52, 67 et 69, à Gil Rémillard, «Tribunaux de première instance : vision d'avenir 》, dans Tribunaux de première instance : vision d'avenir - Actes du colloque 2004, supra note 6 à la p 22 [Rémillard] et à Guy Gagnon, " Ouverture du colloque ", dans Tribunaux de première instance : vision d'avenir-Actes du colloque 2004, supra note 6 à la p 10.

58 Comité de réflexion et d'orientation sur la justice de première instance au Québec, supra note 7 à la page titre (nous soulignons). 
aux besoins des citoyens ${ }^{59}$. Pour y répondre, il opte en conséquence pour une « démarche axée sur les besoins véritables des citoyens », dans un « esprit (...) de véritable préoccupation envers [ces derniers] ${ }^{60}$. Plaidant pour la mise en place d'une cour intégrée de première instance ${ }^{61}$, le Comité tient alors les propos suivants :

« L'organisation hiérarchique actuelle du système judiciaire de première instance doit céder le pas à un système judiciaire moderne, efficient, simplifié et accessible partout sur le territoire ayant comme objectif fondamental de répondre adéquatement aux besoins actuels des justiciables. $»^{62}$

Une réforme structurelle de la justice civile peut également impliquer, non pas l'unification des tribunaux judiciaires existants, mais bien la création de nouvelles instances judiciaires. Ainsi, la Colombie-Britannique a adopté, en mai $2012^{63}$, la loi intitulée Civil Resolution Tribunal Act ${ }^{64}$, laquelle institue le Civil Resolution Tribunal $[\mathrm{CRT}]^{65}$. Le CRT est un nouveau tribunal qui entendra des litiges en matière de copropriété ( « strata disputes ») et, lorsque les parties y consentiront, certains différends impliquant des petites créances ${ }^{66}$. Or derrière la création de ce tribunal, qui devrait entrer en fonction en $2016^{67}$, se trouve l'idée de recentrage. En effet, la mise sur pied de cette nouvelle instance judiciaire représente, pour le gouvernement de Colombie-Britannique, « an attempt to modernize the justice system through a focus on meeting citizen needs, user satisfaction, and continuous improvement through innovation ${ }^{68}$. En d'autres termes, l'on souhaite l'avènement d'un système « focused on users rather than managers $»{ }^{69}$.

Toujours en matière de réorganisation judiciaire, notons enfin que l'idée de recentrage est également venue teinter le débat tenu récemment en France sur ce sujet. En fait foi, notamment, le rapport de la Commission sur la répartition des contentieux, paru en 2008. Dans celui-ci, la Commission, réunie à l'initiative de la ministre de la Justice française, se penche sur la question de l'articulation des

$59 \quad$ Ibid à la $\mathrm{p} 4$.

60 Ibid.

61 Ibid à la $\mathrm{p} 23$.

62 Ibid à la p 22 (nous soulignons).

63 Déposé le 7 mai 2012, le projet de loi a reçu la sanction royale le 31 mai 2012. Voir British Columbia Ministry of Justice, News release, « Online civil dispute tools to save time, money » (7 mai 2012), en ligne, the Province of British Columbia : <http://www2.news.gov.bc.ca/news_releases_2009-2013/2012JAG0068-000600.htm> et « Ministry of Justice Act », en ligne, British Columbia : <http://www.ag.gov.bc.ca/legislation/civil-resolution-tribunal-act/>.

64 PL 44, Civil Resolution Tribunal Act, $4^{\mathrm{e}}$ sess, $39^{\mathrm{e}}$ lég, Colombie-Britannique, 2011-2012 (troisième lecture le 30 mai 2012).

65 Voir le site du Tribunal : < http://www.civilresolutionbc.ca $>$.

66 Dont la valeur est établie en Colombie-Britannique à $25000 \$$ et moins. Voir « When can I use the CRT? », en ligne, CRT : <http://www.civilresolutionbc.ca/what-is-the-crt/when-can-i-use-the-crt/>. Sur le fonctionnement du CRT ainsi que sur sa procédure, voir « How will the CRT work? », en ligne, CRT : <http://www.civilresolutionbc.ca/what-is-thecrt/how-will-the-crt-work/>.

67 «What is the CRT? », en ligne, CRT : <https:/www.civilresolutionbc.ca/what-is-the-crt/>.

68 British Columbia Ministry of Justice, White Paper, supra note 9 à la p 11 (nous soulignons).

69 Ibid. Voir également, pour d'autres affirmations de l'idée de recentrage, les pages 2, 3, 4 et 7. 
contentieux ${ }^{70}$, quelques semaines à peine après la refonte de la carte judiciaire étant intervenue en France $^{71}$. Elle y propose « une rénovation de l'organisation judiciaire de la première instance », et ce, «en partant de l'idée qu'il faut replacer le justiciable au centre du système ${ }^{72}$. Cette dernière idée constitue, selon ses propres dires, sa «préoccupation première $»^{73}$. En introduction de son rapport, la Commission écrit en effet ceci : «Si le juge doit être replacé au cœur de son activité juridictionnelle, le justiciable doit être mis au centre de l'institution judiciaire ${ }^{74}$. Cette " nouvelle philosophie de l'institution judiciaire $»^{75}$ que met de l'avant et adopte la Commission, philosophie qui « met la personne au centre ${ }^{76}$ du système, l'amène notamment à poser l'intérêt du justiciable comme principe directeur sous-tendant ses travaux. Ce principe directeur implique de « se mettre à la place du justiciable », et ce, parce qu' «il appartient à l'administration de s'adapter à l'usager et non l'inverse $»^{77}$.

\section{Modernisation des systèmes de justice civile}

En troisième lieu, la nécessité de penser la justice civile en fonction, premièrement, des besoins du citoyen a également été soulevée dans le domaine plus vaste de la modernisation des systèmes de justice civile. On réfère ici à la réforme de la justice civile comprise dans son sens le plus large, dépassant les questions plus précises de la révision de la procédure civile ainsi que de la refonte de l'organisation judiciaire (qui en sont, en quelque sorte, des sous-ensembles). Comme en matière d'accès à la justice, le

70 Question qui a été abordée largement. Voir Commission sur la répartition des contentieux, supra note 6 à la p 31, ainsi que les détails des objectifs poursuivis par la Commission aux pp 34 et s.

71 Ibid aux pp 3 et 31 .

72 Ibid à la p 43. Soulignons que la seconde partie du rapport est justement intitulée « L'articulation des contentieux. Remettre le justiciable au centre du système judiciaire » (nous soulignons), faisant ressortir du même coup l'importance qu'y occupe l'idée de recentrage.

73 Ibid à la $\mathrm{p} 183$.

74 Ibid aux pp 38-39 (nous soulignons), citant le président de la Commission, Serge Guinchard, lors de son discours d'installation tenu le 18 janvier 2008, discours d'ailleurs intitulé : « Justice au cœur justiciable au centre ». Notons que la Commission affirme que c'est là, également, l'avis « des sociologues et théoriciens de la justice que sont MM. Jacques Commaille et Antoine Garapon », qui « l'ont rappelé à la commission dès les premières auditions. » (ibid). Voir dans le même sens la page $40:$ : (...) les membres de la commission ont gardé à l'esprit l'impérieuse nécessité d'un humanisme judiciaire, le respect des grands principes fondateurs de notre société. Cette nécessité et ces principes les ont conduits à mettre le justiciable au centre du système judiciaire. » (nous soulignons). Cet angle d'approche comporte néanmoins une part de défi : « Le défi posé à la commission n'est pas de passer d'une justice qui se contenterait de gérer des flux et qui, à ce titre fait date, à une justice managériale qui fait peur, mais de passer d'une justice qui trouve sa légitimité exclusivement dans l'application de la loi, à “ une justice plus attentive aux besoins des citoyens, soucieuse d'augmenter leur capacité, de les autoriser à agir "; la justice glisserait ainsi " du modèle classique de l'autorité à celui de l'autorisation ". Il y a là “ une recomposition majeure qui prétend mettre la personne au centre des institutions ${ }^{(3)}$ ". La dynamique se transforme : le sujet devient le juge de ses intérêts ; dans de nouveaux modes alternatifs de résolution des conflits, telle la procédure participative que la commission propose, sa passivité est remplacée par son activité et sa responsabilité. Ce nouveau modèle de justice valorise l'autonomie des citoyens et implique une recomposition du rôle du juge, ainsi qu'une redéfinition de l'ordre public. Dès lors, une justice accessible étant essentiellement orientée vers la satisfaction des besoins des justiciables, son organisation et son fonctionnement doivent placer la personne au centre de $\underline{1}^{\prime}$ institution ${ }^{(1)}$. » (ibid aux pp 42-43; références omises, nous soulignons).

75 Ibid à la p 43 .

76 Ibid.

77 Ibid à la p 183, rapportant des propos de la « Conférence des présidents de TGI » (ibid). 
plaidoyer en faveur d'une $"$ orientation usager ${ }^{78}$ dans le contexte d'une réforme de la justice civile serait une tendance récente ${ }^{79}$ et, au surplus, mondiale ${ }^{80}$. Quant à ce caractère récent, nuançons en rappelant que dans ce domaine, les premières occurrences de l'idée de recentrage remonteraient au début des années $1990^{81}$. Ainsi, par exemple, dans un rapport paru en 1992 et consacré à l'avenir du système de justice de l'État du Massachusetts, on peut lire la phrase suivante, certainement d'avant garde : «In 2022 the Massachusetts justice system will be oriented to its users; (..) $»^{82}$. Cette projection est intéressante étant donné son caractère «affirmatif » : l'idée de recentrage y est posée non pas comme un simple énoncé d'intention, un souhait (« the (...) justice system should be oriented to its users »), mais comme une véritable vision d'avenir en matière de réforme de la justice (« the (...) justice system will be oriented to its users »). C'est donc dire que, dès 1992, l'on jugeait ce recentrage non seulement réaliste, réalisable, mais même, en quelque sorte, inévitable ${ }^{83}$.

Quelques années plus tard, en 1996, c'est au tour de l'Association du Barreau canadien d'embrasser l'idée d'un recadrage de la justice civile autour du citoyen. L'ABC écrit :

« The most important participants in the civil justice system are members of the public, both individuals and organizations, seeking to have disputes resolved. It is for their benefit and that of Canadian society as a whole that the civil justice system was created. It follows that the system should continue to exist, in its current or an adapted form, only so long as it serves the needs of Canadians and is considered by them to be relevant, accessible and fair. ${ }^{84}$

78 Traduction libre de l'expression « user orientation » employée notamment par Alan Uzelac, « Goals of Civil Justice and Civil Procedure in the Contemporary World Global Developments - Towards Harmonisation (and Back) », dans Uzelac, supra note 40 aux pp 5 et 27 [Uzelac, « Goals »]. L’Association du Barreau canadien utilise la même expression dans Canadian Bar Association, Task Force Report, supra note 9 à la p 56.

79 C'est là l'avis, notamment, de Georges De Leval, Le citoyen et la justice civile : un délicat équilibre entre efficacité et qualité, Bruxelles, Bruylant, 2006, à la p 32 [De Leval, « citoyen »]; d'Alan Uzelac (« Goals », supra note 78 aux pp 2728 ) et de Jean-François Roberge, Le sentiment d'accès à la justice et la conférence de règlement à l'amiable. Rapport de recherche sur l'expérience des justiciables et avocats à la Cour supérieure du Québec et à la Cour du Québec, $1^{\mathrm{er}}$ décembre 2014, page iii [Roberge, Le sentiment d'accès à la justice].

80 Roberge, Le sentiment d'accès à la justice, supra note 79 à la p iii.

81 À tout le moins en Amérique du Nord et en Australie, tel que nous l'affirmions ci-dessus (p 4, n 11).

82 Chief Justice's Commission on the Future of the Courts, Reinventing justice, 2022: report of the Chief Justice's Commission on the Future of the Courts, Boston, Supreme Judicial Court, Commonwealth of Massachusetts, 1992, sous « vision » (page non numérotée) [Chief Justice's Commission on the Future of the Courts]. Voir également ibid, à la p 12 : «Every employee, every procedure, every structure in the Massachusetts justice system must put the public and the justice seeker first. " (nous soulignons). Sur l'idée de recentrage qui devrait s'appliquer à toutes les composantes du système, voir également Richard Zorza, The Self-Help Friendly Court: Designed from the Ground Up to Work for People Without Lawyers, Williamsburg, The National Center for State Courts, 2002, aux pp 11, 13 et 18 [Zorza]; Rebecca Love Kourlis et Dirk Olin, Rebuilding justice: civil courts in jeopardy and why you should care, Golden, Fulcrum Pub, 2011, à la p 192 [Kourlis et Olin] et Parker, supra note 11 à la p 15.

83 Ajoutons que cette projection vient également relativiser le caractère novateur des propositions de réforme de la justice civile axées sur un recentrage qui sont avancées aujourd'hui. Cela dit, le fait qu'un recentrage soit, encore aujourd'hui, posé comme projet de réforme en matière de justice civile nous montre également que la vision d'avenir posée aussitôt qu'en 1992 ne s'est toujours pas matérialisée, et donc que le projet conserve toute sa pertinence, demeure d'actualité.

84 Canadian Bar Association, Task Force Report, supra note 9 à la $\mathrm{p} 3$. 
Il s'ensuit que le fait de répondre aux attentes et aux besoins des justiciables devrait être, de l'avis de l'ABC, un des objectifs premiers poursuivis par toute réforme de la justice civile, objectif qui implique de porter une attention particulière à l'usager du système ${ }^{85}$. Pour citer l'Association à nouveau : « (...) the outcome urged by the Task Force is a civil justice system for the twenty-first century that: - is responsive to the needs of users and encourages and values public involvement (..) $\gg^{86}$. L'ABC réitérera ensuite cette position dans son rapport paru en 2013, dont il a été question plus haut ${ }^{87}$.

En guise d'illustration du caractère « international » de l'idée de recentrage appliquée à la réforme de la justice civile, mentionnons qu'elle est avancée dans plusieurs sources récentes parues aux États-Unis. Par exemple, dans leur ouvrage intitulé Rebuilding Justice. Civil Courts in Jeopardy and Why You Should Care $^{88}$, Rebecca Love Kourlis et Dirk Olin écrivent ce qui suit :

« The civil courts exist to serve citizens: parties to current lawsuits, parties to lawsuits to come, (...). Why are citizens not then at the center of every equation, every decision? $(\ldots)$

Our vision is simple: courts that are built for service to the public. In our vision, (...) every procedure would be designed for the person or party seeking justice (...). ${ }^{89}$

Reprenant l'idée de recentrage quelque peu différemment, Victor E. Flango et Thomas M. Clarke plaident pour leur part pour une nécessaire réorganisation du travail des tribunaux à partir d'une conceptualisation de justiciable comme client (« the litigant as a customer ») :

« The starting point for defining the mission of a modern court must be the recognition that courts have customers: the litigants and the public. (...) We argue in this book that courts should respond in three specific ways to the ongoing crisis. First, they must fundamentally reorganize their business processes around the concept of the litigant as a customer. (...) $)^{90}$

85 Ibid à la p $55:$ : One of the primary objectives of civil justice reform should be to increase the responsiveness to the needs and expectations of people who come into contact with the courts. There must be a renewed focus on the individual user (...).»

86 Ibid à la p 7; voir également la page 28. Ici encore, on retrouve formulée une vision d'avenir du système de justice civile; voir à ce propos ibid à la p 23. Notons que la même vision du futur ressort d'un rapport américain paru la même année. Voir Commission on the Future of the Tennessee Judicial System, To Serve All People: a Report, Nashville, Administrative Office of the Courts of Tennessee, 1996, aux pp xiii, 8, 16, 33 et 67 [Commission on the Future of the Tennessee Judicial System].

87 Association du Barreau canadien, Justice pour tous, supra note 5 notamment aux pp 14-15, 65-66 et 104.

88 Kourlis et Olin, supra note 82.

89 Ibid aux pp 191-192.

90 Victor E Flango et Thomas M Clarke, Reimagining Courts: A Design for the Twenty-First Century, Philadelphie, Temple University Press, 2015, aux pp 11-12 [Flango et Clarke]. Voir également la page 13. Sur le justiciable comme client, voir également Commission on the Future of the Tennessee Judicial System, supra note 86 à la p 33. 
L'idée qui nous intéresse est au surplus mise de l'avant en Belgique, notamment par le professeur Georges De Leval. Selon ce dernier, il est nécessaire, dans les discussions portant sur une réforme éventuelle du système de justice civile, de s'assurer que le citoyen soit toujours " au centre du débat », et ce, " non seulement dans les discours, mais surtout dans la réalité $»{ }^{91}$, afin de faire en sorte qu'il ne devienne jamais «le captif d'enjeux qui le dépassent » ${ }^{92}$. Pour ce faire, il propose de « considérer la justice à travers le justiciable et non l'inverse ${ }^{93}$. Cela implique de s'assurer que ce justiciable, « même potentiel » (donc en sa qualité de citoyen), soit « convaincu de ce que (...) ses besoins essentiels sont rencontrés $(\ldots) »^{94}$. On peut faire mention également du cas de la France. Par exemple, dans un livre paru en 2006, les auteurs Matthieu Boissavy et Thomas Clay formulent 110 propositions de réforme qui poursuivent toutes " un même objectif, un même idéal : replacer le citoyen au cœur du système judiciaire car il en est le grand oublié $\gg{ }^{95}$. Pour ces auteurs, la mise du justiciable tout à fait au centre des préoccupations de l'institution judiciaire constitue ni plus ni moins que le « grand défi d'une justice moderne $\gg{ }^{96}$.

L'appel de Boissavy et Clay semble avoir été entendu en France puisque, en 2014, un grand débat national sur la réforme de l'institution judiciaire s'est tenu à la maison de l'UNESCO à Paris. Un événement qui a réuni pas moins de 1900 personnes $^{97}$ et lors duquel l'idée de recentrage a occupé une place centrale. À cet égard, le titre donné à ce débat public est éloquent : « La Justice du $21^{\mathrm{e}}$ siècle : Le citoyen au cœur du service public de la justice ${ }^{98}$. L'idée de recadrer la justice civile autour de l'usager ressort également très clairement du programme de ces journées :

«Le Président de la République, dans son allocution lors de l'audience solennelle de la Cour de cassation, le 18 janvier 2013, a fait de la justice de proximité une de ses priorités pour la Justice. D'abord une proximité géographique, permettant à la justice d'être au plus proche des citoyens et de leurs besoins ; ensuite, une proximité concrète permettant d'assurer au citoyen une réponse effective, efficace et efficiente à ses demandes ; enfin, une proximité personnelle, lui permettant de mieux comprendre le processus judiciaire et d'être l'acteur de ses droits.

C'est la raison pour laquelle Christiane Taubira, garde des sceaux, ministre de la justice a souhaité, au-delà de certaines mesures urgentes et immédiates nécessaires pour répondre

91 De Leval, « citoyen », supra note 79 à la p 10. Voir dans le même sens les pages 40 et 45-46.

92 Ibid à la p 10. Voir également la page 205.

93 Ibid à la $\mathrm{p} 30$.

94 Ibid à la p 40.

95 Boissavy et Clay, supra note 46 à la p 20. Voir aussi les pages 21, 27, 28, 29, 79-80, 83, 91,95 et 108.

96 Ibid à la p 80.

97 Ces 1900 personnes comptaient des magistrats, des fonctionnaires de justice, des professionnels du droit, des universitaires, des parlementaires ainsi que des élus locaux. Voir à ce propos Projet de loi portant application des mesures relatives à la justice du XXI ème siècle (PROCÉDURE ACCÉLÉRÉE), texte n 661 (2014-2015) présenté au nom de M. Manuel VALLS, Premier ministre, par $\mathrm{M}^{\mathrm{me}}$ Christiane TAUBIRA, Garde des Sceaux et ministre de la Justice, déposé au Sénat le 31 juillet 2015, à la p 6 de l'exposé des motifs, en ligne, Sénat : <http://www.senat.fr/leg/pj114661.pdf $>$ [projet de loi 661].

98 La Justice du $21^{e}$ siècle : Le citoyen au cœur du service public de la justice-Les actes du débat national, 10-11 janvier 2014, Maison de l'UNESCO, page titre, en ligne, ministère de la Justice : < http://www.justice.gouv.fr/publication/j21_actes.pdf $>$ [La Justice du $21^{e}$ siècle, les actes du débat national]. 
à la difficile situation des juridictions, conduire plusieurs réflexions pour placer le citoyen au cour des réformes de la Justice. ${ }^{99}$

Dans les mots de la ministre, l'objectif du débat était de " dégager les axes d'une réforme judiciaire tournée vers le service rendu au justiciable et qui (...) doit permettre un projet de société, c'est-à-dire un projet qui s'adresse à l'ensemble des citoyens ${ }^{100}$. Dans cet esprit, $\mathrm{M}^{\mathrm{me}}$ Taubira a formé un Comité de pilotage des réformes de justice chargé de présider le débat et de dégager, à son issue, des pistes de réflexion à être soumises ensuite aux divers acteurs touchés par les réformes ${ }^{101}$. Soulignons que dans le discours qu'il a prononcé à l'ouverture du débat, le premier ministre français de l'époque, M. Jean-Marc Ayrault, a lui aussi fait écho à l'idée d'une justice tout entière tournée vers le citoyen, en affirmant notamment ce qui suit : « le cour du métier, au-delà de l'acte de juger, c'est le service aux citoyens. » ${ }^{102}$

En sus de ces deux journées de débat public, des consultations ont eu lieu au sein des différents ressorts français, consultations qui ont associé magistrats, fonctionnaires et partenaires de la justice. Il en a résulté plus de 2000 contributions d'acteurs de justice de terrain ${ }^{103}$. Puis, le 10 septembre 2014, la Garde des Sceaux a présenté en Conseil des ministres quinze « actions pour la justice du XXI ${ }^{\text {ème }}$ siècle », lesquelles visaient à " garantir un service public de la justice plus proche, plus efficace et plus protecteur ${ }^{104}$. Concrètement, la mise en œuvre de ces actions a pris la forme, jusqu'à présent, de divers textes juridiques ${ }^{105}$. En fait partie le Projet de loi portant application des mesures relatives à la justice

99 Voir « Programmes des journées », en ligne, ministère de la Justice : $<$ http://www.justice.gouv.fr/la-justice-du-21esiecle-12563/10-et-11-janvier-2014-debats-publics-12748/programme-des-journees-27587.html> (nous soulignons). Notons que cet événement fait suite au dépôt de quatre rapports auprès de la ministre, rapports s'inscrivant dans le cadre de la même initiative et qui avaient pour objet d'orienter les réflexions. Ceux-ci sont disponibles à « Groupes et commissions de travail », en ligne, ministère de la Justice : <http://www.justice.gouv.fr/la-justice-du-21e-siecle12563/10-et-11-janvier-2014-debats-publics-12748/groupes-et-commission-de-travail-27582.html>.

100 La justice du $21^{e}$ siècle - le citoyen au cour de la justice. Les scénarios de réforme, page 3, en ligne, ministère de la Justice : <http://www.justice.gouv.fr/publication/j21scenarios_reforme.pdf $>$ [La justice du $21^{e}$ siècle, les scénarios de réforme].

101 La Justice du $21^{e}$ siècle, les actes du débat national, supra note 98 aux pp 7 et 8.

102 Voir « Ouverture. Discours de Monsieur Jean-Marc Ayrault, Premier Ministre », dans La Justice du $21^{e}$ siècle, les actes du débat national, supra note 98 à la p 14.

103 Voir Étude d'impact, Projet de loi portant application des mesures relatives à la justice du XXI $I^{\text {eme }}$ siècle, NOR : JUSX1515639L, 31 juillet 2015, à la p 7, en ligne, Sénat : <http://www.senat.fr/leg/etudes-impact/pj114-661-ei/pj114661-ei.pdf> [Étude d'impact].

104 Projet de loi no 661, supra note 97 à la p 7. Ces quinze actions sont les suivantes : Faciliter les démarches des citoyens dans toutes les juridictions; Faciliter les démarches des justiciables sur internet grâce à « Portalis »; Renforcer la politique d'accès au droit; Évaluer, au niveau local, les besoins de droit et de justice en association avec la société civile; Ouvrir le service public de la justice sur la société par la création de conseils de juridiction; Simplifier les délais, les règles et les recours en matière civile; Favoriser les modes alternatifs de règlement des litiges; Permettre aux citoyens de mieux évaluer les possibilités de succès de leurs actions en justice; Recentrer les magistrats sur leur cœur de métier; Réorganiser les parquets et améliorer les méthodes de travail pour leur permettre de mieux accomplir leurs missions; Renforcer la communauté de travail dans les juridictions; Intensifier la politique d'aide aux victimes; Recentrer le tribunal d'instance sur la justice du quotidien et sur la protection des personnes vulnérables; Sécuriser la vie économique et enfin améliorer le statut des magistrats pour mieux garantir les libertés. Voir Étude d'impact, supra note 103 aux pp 7 8.

105 Ibid à la p 8 . 
$d u X X I^{e ̀ m e}$ siècle $^{106}$ (ci-après appelé le « projet de loi $\left.661 »\right)$ déposé au Sénat par $\mathrm{M}^{\text {me }}$ Taubira le 31 juillet 2015. Comme il fallait s'en douter, l'idée de recentrage y est centrale. L'exposé des motifs qui ouvre le projet de loi est, à ce propos, on ne peut plus clair. On y lit entre autres que le texte législatif comporte sept titres qui ont pour finalité «d'améliorer la justice du quotidien et de placer le citoyen au cœur du service public de la justice ${ }^{107}$. On y retrouve, au surplus, le passage suivant :

« La justice doit être en phase avec les évolutions de la société et davantage tournée vers le citoyen pour répondre à ses nombreuses attentes et lui permettre d'être plus en capacité d'agir pour défendre ses droits et résoudre ses litiges. Il nous faut adapter l'organisation et le fonctionnement de la justice pour que le citoyen soit au cœur du service public de la justice. $\gg{ }^{108}$

Les développements qui précèdent ont montré que l'idée de «placer le citoyen au cœur du système » occupe une place de choix dans les discussions entourant la question de la réforme de ce système, et ce, internationalement. Une réforme comprise ici, on l'a dit, largement, visant autant la révision de la procédure civile que la refonte de l'organisation judiciaire et la modernisation de la justice. Nous verrons maintenant en quoi cette idée est également avancée non plus dans le contexte de la réforme de la justice civile, mais plutôt dans celui de son évaluation.

\section{L'évaluation de la justice civile}

Si l'on rencontre l'idée de recadrage de la justice civile autour du citoyen dans la documentation portant sur l'accès à la justice civile ainsi que dans celle abordant sa réforme, c'est également le cas dans les sources traitant de son évaluation, notamment au plan qualitatif. Le domaine de l'évaluation de la qualité de la justice civile constitue donc le troisième contexte d'émergence de l'idée de recentrage. Précisons d'emblée que, paradoxalement, la documentation consultée révèle que l'évaluation de cette qualité peut s'entendre quantitativement, en désignant alors le recours - du reste de plus en plus fréquent $^{109}$ - à des indicateurs chiffrés en matière de justice ${ }^{110}$. Prise en ce sens, l'idée d'évaluer la qualité de la justice civile s'inscrirait dans le mouvement plus général de la réforme de l'État et viserait à

106 Pour la référence complète de ce projet de loi, voir supra note 97.

107 Projet de loi 661, supra note 97 à la p 7 (nous soulignons).

108 Ibid à la p 6. Voir également la p 8 : «Afin de placer le citoyen au cœur du service public de la justice, il faut tout d'abord rapprocher la justice du citoyen en garantissant mieux l'accès au droit et à la justice, garantie fondamentale que l'État se doit d'assurer dans une démocratie (...) ».

109 Il s'agirait, par exemple, d'une tendance en Europe. Sur un tel constat, voir entre autres Commission européenne pour l'efficacité de la justice (CEPEJ), Systèmes judiciaires européens, Édition 2010 (données 2008) : Efficacité et qualité de la justice, Éditions du Conseil de l'Europe, Strasbourg, 2010, aux pp 104 et 113 [CEPEJ] et Gar Yein Ng, « A discipline of judicial governance ? », (2011) 7 Utrecht LR 102, à la p 112. Notons que l'évaluation de la qualité comme tendance européenne dépasse le domaine de la justice, pour concerner plus généralement celui de l'action publique, donc de tous les services publics. Voir Hélène Pauliat, « La qualité : une panacée, une mode ou une politique cohérente au plan européen ? », dans Pauliat, supra note 4 à la p 206 [Pauliat, « la qualité »].

110 Sur cette appréciation « quantitative » de la qualité de la justice, voir entre autres Cécile Castaing, «Les procédures civile et administrative confrontées aux mêmes exigences du management de la justice », (2009) AJDA 913, aux pp 2, 7 et 10 [Castaing]; Loïc Cadiet, « Case management judiciaire et déformalisation de la procédure », (2008) 125 R française d'administration publique 133, à la p 135 [Cadiet], et Boissavy et Clay, supra note 46 aux pp 77 et 80-82. 
répondre aux impératifs liés à ce que l'on a appelé la «nouvelle gestion publique ${ }^{111}$ (new public management $)^{112}$. Selon Antoine Garapon, cette nouvelle gestion publique a préfiguré l'émergence d'un nouveau modèle de justice, la « justice managériale ${ }^{113}$, qui prendrait racine dans le néolibéralisme.

Bien qu'appelant d'abord l'évaluation de la justice à l'aune du critère d'efficience économique ${ }^{114}$, lequel « me[t] l'accent sur le rendement, le productivisme et l'efficacité de l'exercice de la fonction juridictionnelle ${ }^{115}$ et aurait, partant, insufflé une véritable « culture de performance et de résultat ${ }^{116}$ au sein des systèmes judiciaires, la nouvelle gestion publique se serait également soldée par un « virage client $\gg{ }^{117}$ en matière de justice civile ${ }^{118}$. En témoigne, par exemple, le mandat confié à la Commission Européenne pour l'Efficacité de la Justice (CEPEJ), établie en 2002 par le Comité des Ministres du Conseil de l'Europe. En vertu de ce mandat, la CEPEJ est notamment chargée de « veiller à ce que les politiques publiques en matière judiciaire tiennent compte des usagers de la justice $(\ldots) »^{119}$. Le niveau de satisfaction et de confiance de ces usagers compte ainsi parmi les indicateurs retenus par la Commission pour mesurer la qualité de la justice ${ }^{120}$. Qui plus est, le rapport de la CEPEJ de 2010 révèle que, en Europe :

«Une attention croissante est portée aux attentes et besoins des usagers des tribunaux. L'introduction et l'utilisation d'instruments spécifiques (enquêtes) se développe ainsi dans les pays européens pour évaluer le niveau de satisfaction et de confiance des usagers

111 La « nouvelle gestion publique » aurait vu le jour dans les années 1970 et aurait vocation à être « un instrument conceptuel de modernisation de l'organisation et de la gestion de l'administration publique, dont l'aspect principal concerne la qualité au moindre coût. Le new public management accorde donc une place essentielle aux résultats, notamment financiers. » (Castaing, supra note 110 à la p 1). Sur cette nouvelle gestion publique, voir également ibid à la p 136.

112 Dolorès Grossemy, «Virage client de la justice et évaluation de sa qualité », dans André Riendeau, dir, Dire le droit : pour qui et à quel prix?, Montréal, Wilson \& Lafleur, 2005, 141, à la p 143 [Grossemy].

113 Pour une description ainsi qu'une critique de ce nouveau modèle de justice, voir Antoine Garapon, La Raison du moindre État. Le néolibéralisme et la justice, Paris, Odile Jacob, 2010, aux pp 45 et s [Garapon, La Raison du moindre État]. Cette critique rejoint en partie celle de Loïc Cadiet, qui dénonce pour sa part la « marchandisation » et la « marchéisation » de la justice (Cadiet, supra note 110 entre autres aux pp 136 et 138-139) ainsi que celle de Cécile Castaing, qui parle d'une justice dorénavant commandée par l'idéologie du marché (Castaing, supra note 110 à la p 2; voir aussi les pages 1, 7 et 10). On peut citer aussi Boissavy et Clay, supra note 46 aux pp 28 et 78.

Au sujet de l'efficience économique, voir notamment Richard A Posner, « An Economic Approach to Legal Procedure and Judicial Administration », (1973) 2 J Leg Stud 399, à la p 400 et Hector Fix-Fierro, Courts, Justice and Efficiency: A Socio-Legal Study of Economic Rationality in Adjudication, Oxford, Hart Publishing, 2003, aux pp 27 et 28.

115 Castaing, supra note 110 à la p 1 . Voir aussi la page 2.

116 Ibid à la p 10. Pour Antoine Garapon, cette performance, objectivement mesurée, est aujourd'hui devenue la seule politique en matière de justice, à une époque où la réduction de la dépense publique est érigée en fin en soi. Voir Antoine Garapon, «Le nécessaire retour du choix politique », (2011) 36 Dr \& Justice 1, à la p 2.

L'expression est notamment employée par Grossemy, supra note 112 à la p 141.

118 Voir par exemple l'avis en ce sens de Castaing, supra note 110 à la p 10 et de Grossemy, supra note 112 à la p 141.

119 CEPEJ, supra note 109 à la p 5.

120 Ibid à la p 84 . 
dans les tribunaux. Dans de nombreux pays, il est devenu habituel de réaliser régulièrement de telles enquêtes au niveau national et au niveau des tribunaux. ${ }^{121}$

Pour Antoine Garapon, il y a là une évolution majeure, celle du « déplacement du centre de gravité de l'institution vers l'individu ${ }^{122}$, laquelle a eu, entre autres effets, celui de transformer la justice en un service $^{123}$ et de l'éloigner de sa qualité de pouvoir ${ }^{124}$. Garapon s'exprime en ces termes :

«Alors qu'autrefois la justice ne se souciait pas de rechercher l'adhésion des justiciables puisqu'elle s'autorisait de l'application de la loi commune, voici qu'elle doit se légitimer à présent par l'effectivité du service qu'elle rend aux individus. L'institution doit être orientée non seulement vers la satisfaction des besoins des justiciables, mais elle doit aussi augmenter leur capacité d'agir, leur permettre de se réaliser, d'atteindre des objectifs. ${ }^{125}$

Si Garapon, avec d'autres ${ }^{126}$, se montre assez critique face à ce qu'il appelle le " tournant individualiste $\gg{ }^{127}$ de la justice, d'autres sont plus favorables à la considération nouvelle accordée au justiciable à l'occasion de l'évaluation de la qualité de la justice civile. Ce sont chez ces derniers auteurs que l'on trouvera les formulations les plus claires de l'idée de recentrage. Par exemple, pour Matthieu Boissavy et Thomas Clay, « [1]a quête d'une amélioration qualitative de la justice, avec l'établissement de critères d'évaluation et la mise en place d'un contrôle citoyen, permet de replacer le justiciable au cœur de la justice et de renforcer son adhésion à notre système judiciaire. ${ }^{128}$

121 Ibid à la p 87. On apprend également, dans ce même rapport, que la satisfaction des usagers quant aux services rendus par les tribunaux est posée comme priorité au Danemark, en Espagne, en Suisse, en Irlande du Nord et en Écosse; voir ibid à la p 106. Il est intéressant de faire ressortir le contraste entre cette situation et celle du Canada, où l'on « n'est guère porté à mesurer de façon continue le taux de satisfaction des clients ou utilisateurs à l'égard de leur expérience, puis à en tirer des enseignements » (Association du Barreau canadien, Justice pour tous, supra note 5 à la p 28; voir dans le même sens la p 163).

122 Garapon, La Raison du moindre État, supra note 113 à la p 46.

123 Ibid.

124 Sur ce passage de la justice comme pouvoir à la justice comme service, voir également Guy Canivet, « Économie de la justice et procès équitable », (2001) 46 La Semaine Juridique Édition Générale I 361, à la p 1, et Castaing, supra note 110 à la $\mathrm{p} 7$.

125 Garapon, La Raison du moindre État, supra note 113 à la p 48. Voir également la page 54, où il parle d'une justice devenue « un bien de consommation courante », ce qui ne devrait pas être, dans la mesure où elle n'est pas un bien comme un autre (ibid à la p 65).

126 Pour Cécile Vigour par exemple, cela tient du consumérisme, lequel « marquerait (...) la “ définition du système [judiciaire] comme une industrie de service concernée par sa clientèle plutôt que comme un appareil constitutionnel de régulation publique " (référence omise; voir Cécile Vigour, « Temps judiciaire et logique gestionnaire. Tensions autour des instruments d'action et de mesure », synthèse du rapport scientifique d'une recherche financée par le GIP Mission de recherche Droit \& Justice, décembre 2011, à la p 3). Voir également, pour une critique du justiciable devenu client et de la « nouvelle réthorique de l'usager de la justice » qui s'impose, Cadiet, supra note 110 aux pp 135 et 136 . La critique de la réthorique de l'usager est aussi présentée dans Gaboriau, supra note 4.

127 Garapon, La Raison du moindre État, supra note 113 à la p 46. Sur le justiciable ramené à un simple consommateur, voir également les pages 64-65.

128 Boissavy et Clay, supra note 46 à la p 91 (nous soulignons). Voir dans le même sens la page 79. 
Notons en terminant que l'idée de recentrage est également défendue dans le contexte plus large de la recherche de la qualité de tout service public, quel qu'il soit. Ainsi, selon Hélène Pauliat, « il ne peut y avoir de qualité sans usager », ce qui implique de faire du bénéficiaire ou de l'utilisateur " la figure essentielle de la qualité, de la véritable qualité $(\ldots) »^{129}$. La professeure de droit public plaide ainsi pour « (...) un repositionnement des acteurs au sein des structures administratives, en plaçant le citoyen au centre du processus, non pas de manière figée comme dans les premières politiques de modernisation, mais de manière dynamique, en lui reconnaissant un rôle (...) majeur, d'acteur de l'action publique. $)^{130}$

Toujours dans le contexte de la quête de qualité appliquée à l'action publique en général, Pierre Bauby écrit ceci :
«À l'évidence, cette problématique impose de transformer profondément la perspective pour remettre au centre le consommateur, l'usager et surtout le citoyen.
Le service d'intérêt général n'a aucun sens par lui-même ou pour lui-même, mais uniquement pour répondre aux besoins des destinataires du service, dans toutes leurs diversités.
Dans cette conception, le citoyen, avec toutes les figures qu'il prend, devient le centre, le moteur du service et en particulier de la qualité. (...)
Ainsi pourra-t-on redonner sens au service public ou d'intérêt général : répondre aux besoins des citoyens et de la collectivité. ॥» 131

Les deux auteurs précités en appellent à une meilleure prise en considération du citoyen à l'occasion de l'évaluation de la qualité d'un service public. Ils plaident au surplus pour l'association véritable de ce citoyen à cette démarche d'évaluation ${ }^{132}$, laquelle serait, encore aujourd'hui, trop souvent menée sans lui, « tournée vers l'interne ${ }^{133}$. Il s'agit donc de faire du citoyen un partenaire réel de cette entreprise, ce qui implique de lui donner une voix, de le faire participer au processus évaluatif ${ }^{134}$.

\section{En guise de conclusion : quelques remarques sur les tenants du recentrage}

Nous conclurons cette première partie par quelques observations générales portant sur l'identité des tenants d'un recadrage de la justice civile autour de la personne du citoyen. L'Observatoire du droit à la justice, que nous citions en introduction, compte parmi les nombreux observateurs du monde judiciaire à s'être prononcés en faveur d'un recentrage. Par " observateurs ", nous entendons autant des organisations ou regroupements œuvrant dans le domaine de la justice civile ${ }^{135}$ que des professeurs de

129 Pauliat, « la qualité », supra note 109 à la p 205.

130 Ibid à la p 211 (nous soulignons). Voir dans le même sens les pages 210, 212, 213, 214, 216 et 219.

131 Bauby, supra note 4 aux pp 106-107.

132 Ibid aux pp 103-104 et Pauliat, « la qualité », supra note 109 notamment aux pp 209-210 et 218-219.

133 Pauliat, « la qualité », supra note 109 à la p 209.

134 Pour la même idée soutenue en matière judiciaire plus spécifiquement, voir par exemple Boissavy et Clay, supra note 46 à la p 89. Cela implique, notamment, un recours accru aux méthodes d'évaluation qualitatives, puisque « le contentieux ne peut se résumer à une affaire de chiffres » (Castaing, supra note 110 à la p 7). Voir également, sur cet avis, Garapon, La Raison du moindre État, supra note 113 à la p 57, et Boissavy et Clay, supra note 46 à la p 79.

135 Outre l'Observatoire du droit à la justice, nous pensons ici, par exemple, au Hague Institute for the Internationalisation of Law (HiiL), mentionné plus haut. Voir à ce propos Barendrecht et al, supra note 12. 
droit $^{136}$, s'exprimant à l'occasion de conférences ou par leurs écrits, ainsi que des spécialistes de l'administration judiciaire ${ }^{137}$. Aux voix de ces observateurs s'ajoutent celles d'acteurs du monde judiciaire qui, eux aussi, ont été nombreux à prôner l'idée d'un recentrage. Parmi ceux-ci figurent d'abord des avocats, parfois regroupés en corps professionnels. Nous pensons ici à l'Association du Barreau canadien, dont il a été question précédemment. Rappelons que par deux fois - d'abord en $1996^{138}$, puis tout récemment, en $2013^{139}$-, l'ABC s'est prononcée clairement en faveur de l'émergence d'un système de justice " axé sur les personnes ${ }^{140}$. Plusieurs juges ont également plaidé pour la mise du citoyen au centre du système de justice civile, s'exprimant parfois en leur nom personnel ${ }^{141}$, parfois en comité $^{142}$. Toujours au chapitre des acteurs du monde judiciaire, l'idée de placer le citoyen au cœur du système de justice civile ressort également de nombreux rapports préparés par des commissions et comités mis sur pied par le gouvernement, ici comme ailleurs. Pensons par exemple, pour le cas du Québec, au Comité de révision de la procédure civile de 1998 et, pour celui de la France, à la Commission sur la répartition des contentieux ainsi qu'au Comité de pilotage des réformes de la justice, tous trois mentionnés précédemment. Enfin, on compte aussi, parmi les acteurs du monde judiciaire favorables à un éventuel recadrage de la justice civile autour du citoyen, plusieurs décideurs en matière de justice. Nous référons ici aux ministres de la Justice qui, au fil des ans, pendant qu'ils étaient en fonction ou encore par suite de leur mandat, ont appuyé l'idée d'un recentrage ou, à tout le moins, ont plaidé pour une meilleure prise en considération des attentes et des besoins des citoyens en matière de justice civile. Parmi ceux-ci, on peut mentionner, au Québec, Jérôme Choquette ${ }^{143}$, Serge Ménard ${ }^{144}$,

136 Citons, entre autres, les professeurs Pierre Noreau (supra note 6), Pierre-Claude Lafond (L'accès à la justice civile, supra note 9), Jean-François Roberge (par exemple dans La justice participative, supra note 31) et Hubert Reid (« Le justiciable est-il toujours au centre des réformes de la justice civile ? », dans Noreau, supra note 12 notamment aux pp 56 et 57 [Reid]). Hors Québec, citons encore le professeur italien Mauro Cappelletti (« Access to Justice as a Theoretical Approach to Law and a Practical Programme for Reform », (1992) 109 S African LJ 22, à la p 57), le professeur belge Georges De Leval (« citoyen », supra note 79), les professeurs français Serge Guinchard (ayant agi comme président de la Commission sur la répartition des contentieux dont il a été question précédemment; voir supra note 6), Hélène Pauliat (« la qualité », supra note 109) et Thomas Clay (supra note 46), le professeur croate Alan Uzelac (« upside down », supra note 40 et « Goals » supra note 78) et le professeur australien Stephen Parker (supra note 11).

Nous pensons ici, notamment, à Victor E. Flango et Thomas M. Clarke, dont le livre (supra note 90) prend racine dans leur expérience respective au National Center for State Courts (NCSC). Au sujet du NCSC, voir son site Web : $<$ http://www.ncsc.org/About-us.aspx $>$.

138 Canadian Bar Association, Task Force Report, supra note 9, notamment aux pp 3 et 55.

139 Association du Barreau canadien, Justice pour tous, supra note 5, notamment aux pp 14-15, 65, 66 et 104.

140 Ibid aux pp 14-15.

141 Citons ici, notamment, l'honorable Beverley MacLachlin, juge en chef de la Cour suprême du Canada : « (...) il est devenu manifeste que nous échouions de plus en plus dans notre devoir de fournir un système de justice accessible qui réponde aux attentes des citoyens et qui soit axé sur ceux-ci. » (nous soulignons). Voir Comité d'action sur l'accès à la justice en matière civile et familiale, feuille de route, supra note 7 à la p i. Mentionnons également l'avis en ce sens de Martine Compte, Première Présidente de la cour d'appel d'Orléans, rapporté dans La Justice du 21e siècle, les actes du débat national, supra note 98 à la p 10. L'on peut également souligner le cas d'anciens juges s'étant prononcés en faveur d'un recentrage, dont fait par exemple partie Rebecca Love Kourlis (supra note 82).

142 Lesquels comités ne sont pas forcément composés uniquement de juges, précisons-le. Outre le Comité d'action sur l'accès à la justice en matière civile et familiale, mentionnons par exemple le Comité de réflexion et d'orientation sur la justice de première instance au Québec, la « Commission on the Future of the Tennessee Judicial System » ainsi que le «Chief Justice's Commission on the Future of the Courts » du Massachusetts, tous cités précédemment.

143 Choquette, supra note 43. 
Paul Bégin ${ }^{145}$ et Gil Rémillard ${ }^{146}$, en Colombie-Britannique, Shirley Bond ${ }^{147}$ et, en France, Christiane Taubira ${ }^{148}$.

L'objectif de ce rapide tour d'horizon des défenseurs de l'idée de recentrage est d'abord d'attirer l'attention sur leur nombre, pour marquer le fait que cette idée ne constitue pas un message isolé, véhiculé uniquement par une poignée de convaincus. Nous voulons aussi rendre compte de leur diversité. L'idée de placer le citoyen au cœur du système n'émane pas seulement du cercle des observateurs du monde judiciaire, peut-être plus facilement critiques du système et de ses dirigeants. Elle compte aussi, parmi ses adhérents, des acteurs de la justice et, à fortiori, des décideurs en la matière. Or, à notre avis, le fait que l'idée de considérer la justice civile à travers le prisme du citoyen soit relayée à l'échelle des décideurs politiques a pour effet de venir, en quelque sorte, l'" officialiser », son poids s'en trouvant, dès lors, augmenté. Ajoutons que ces observateurs, acteurs et décideurs sont issus de juridictions diverses : du Canada (par exemple du Québec, de la Colombie-Britannique et de l'Ontario), des États-Unis, de l'Australie, de la France, de la Belgique, de l'Italie, de la Chine, du Brésil et de la Croatie, et des recherches supplémentaires en révéleraient certainement d'autres, puisque l'idée de recentrage constitue, comme nous le soulignions plus haut, une tendance internationale. Par conséquent, cette idée n'est pas circonscrite géographiquement, elle ne semble pas propre à une culture juridique particulière. Ici encore, le fait qu'elle traverse les frontières et transcende les particularismes propres aux systèmes judiciaires nationaux nous semble lui conférer un intérêt accru et justifier que l'on s'y arrête.

\section{LES FONDEMENTS JUSTIFICATIFS DE L'IDÉE DE RECENTRAGE}

Nous nous pencherons maintenant sur les fondements justificatifs de l'idée de recentrage. La question qui nous intéresse ici est celle de savoir pourquoi les tenants d'un recentrage plaident pour celui-ci, quels sont les motifs qu'ils avancent à l'appui de leur position ou qui se dégagent implicitement de leurs propos. Avant d'y venir, une remarque préliminaire s'impose. L'idée de recentrage ne fait pas l'objet d'un discours parfaitement homogène, unifié. En effet, l'argumentaire en faveur d'une mise du citoyen au centre du système de justice civile a été, on l'a vu, avancé par des personnes différentes, dans des contextes divers, ainsi que dans des juridictions tout aussi diverses. Il s'ensuit que tous les tenants du recentrage n'ont pas pu, forcément, justifier leur position exactement de la même manière. Conséquemment, nous ne prétendons pas, dans la présente partie, rendre compte de l'existence d'une justification unique de l'idée de recentrage.

Cela étant dit, au regard de la documentation consultée, il est tout de même possible de dégager un certain nombre de fondements justificatifs qui reviennent dans la très grande majorité des sources dans lesquelles l'idée de recentrage est défendue. Ce qui suit relève donc d'une synthèse des justifications les

144 Au regard des termes du mandat confié en 1998 au Comité de révision de la procédure civile. Voir ci-dessus (page 12).

145 Voir les propos du ministre rapportés dans Beaudoin, supra note 6.

146 Voir Rémillard, supra note 57 à la p 22 (se prononçant ici après son mandat, lequel s'est échelonné du 23 juin 1988 au 11 janvier 1994). Mentionnons également l'article signé par Michel Bouchard en 1999 (également évoqué précédemment), pendant qu'il était sous-ministre de la Justice et sous-procureur général du Québec. Voir Bouchard, supra note 6 aux pp 8-9.

147 Voir le message introductif de la ministre contenu dans British Columbia Ministry of Justice, White Paper, supra note 9 à la p 2.

148 Voir La justice du $21^{e}$ siècle, les scénarios de réforme, supra note 100. 
plus fréquemment avancées à l'appui de l'idée d'un recadrage de la justice civile autour du citoyen. Nous exposerons d'abord la finalité première de la justice civile telle que la conçoivent les tenants d'un recentrage (A), avant de nous pencher sur l'avis, partagé par ceux-ci, selon lequel la justice civile se serait, aujourd'hui, écartée de cette vocation principale (B).

\section{A. La justice civile : un service public destiné au citoyen}

Pourquoi faut-il mettre le citoyen au cœur du système de justice civile ? Parce que cette justice civile serait, d'abord et avant tout, un service public ${ }^{149}$. Il s'agirait là de sa nature profonde, de sa finalité, sa vocation première. Toute l'existence du système judiciaire reposerait sur cette mission fondamentale ${ }^{150}$. Autrement dit, le système de justice civile aurait été créé, conçu, d'abord pour le citoyen ${ }^{151}$. Il est intéressant de constater que les auteurs adhérant à cette vision ou cette conception du système de justice civile sont à ce point convaincus qu'ils l'énoncent souvent comme un rappel à l'intention des acteurs du monde judiciaire, qui l'auraient oublié ou perdu de vue. C'est le cas, notamment, du Comité d'action sur l'accès à la justice en matière civile et familiale, qui affirme ce qui suit dans sa Feuille de route pour le changement: "Ceux d'entre nous qui travaillent à l'intérieur du système doivent se rappeler que le système existe pour desservir le public. C'est sur cela que nous devons axer tous nos efforts de réforme. ${ }^{152}$

149 Sur un tel constat, voir notamment Bouchard, supra note 6 à la p 9; Rémillard, supra note 57 à la p 22; De Leval, " citoyen », supra note 79 aux pp 45-46; Comité d'action sur l'accès à la justice en matière civile et familiale, feuille de route, supra note 7 aux pp iii, iv, 1 et 8; Comité de révision de la procédure civile, supra note 6 aux pp 31, 33, 36 et 38; Noreau, «Avenir de l'institution judiciaire », supra note 6 à la p 67; Association du Barreau canadien, Justice pour tous, supra note 5 aux pp 14-15; Noreau, « justice », supra note 6 à la p 48; Lachapelle et al, « solutions », supra note 1; Roberge, "perspectives », supra note 28 aux pp 18 et 27; Action committee on access to justice in civil and family matters, Prevention, triage and referral working group, supra note 12 aux pp i et 35; Observatoire du droit à la justice, supra note 54 aux pp 9 et 57; Kourlis et Olin, supra note 82 aux pp 187 et 192, et Boissavy et Clay, supra note 46 entre autres aux pp 20 et 26. Notons que certains parlent également de la justice civile comme d'un « bien public» (Association du Barreau canadien, Justice pour tous, supra note 5 à la p 93), ou encore d'un «bien commun » (Observatoire du droit à la justice, supra note 54 à la p 56). L'honorable Beverley McLachlin en fait pour sa part un « bien social de base » (voir ses propos rapportés dans Buzzetti, supra note $6:$ : Comment considérons-nous la justice? S'agit-il d'un bien de base qu'une société civilisée se doit de procurer à ses membres? Ou s'agit-il d'un bien de luxe, (...) que seuls peuvent s'offrir ceux qui en ont les moyens, mais pas les autres? » La juge avait répondu à sa propre question réthorique. «La justice est un bien social de base, tout comme la nourriture, le logement et les soins médicaux.»).

150 Voir par exemple Rémillard, supra note 57 à la p 22; Comité d'action sur l'accès à la justice en matière civile et familiale, feuille de route, supra note 7 à la p 8; Canadian Bar Association, Task Force Report, supra note 9 aux pp 3 et 19.

151 Voir entre autres Lafond, L'accès à la justice civile, supra note 9 à la p 7; Buzzetti, supra note 6; Canadian Bar Association, Task Force Report, supra note 9 à la p 3 et Boissavy et Clay, supra note 46 à la p 23.

152 Comité d'action sur l'accès à la justice en matière civile et familiale, feuille de route, supra note 7 à la $\mathrm{p} 8$. Voir également Boissavy et Clay, supra note 46 à la p 23 : «Faut-il rappeler que la justice n'est pas faite pour les magistrats, ni pour les auxiliaires de justice, mais pour les justiciables? ». Voir, dans le même sens, Lachapelle et al, « solutions », supra note 1, et Buzzetti, supra note 6, pour cette affirmation posée en évidence : «L'essentiel est de remettre le citoyen au cœur du système de justice pour qui, après tout, il est conçu. » (nous soulignons). Sur l'idée connexe voulant que cette mission de service public est souvent tenue pour acquise, voir Commission on the Future of the Tennessee Judicial System, supra note 86 à la p xiii. Parker souligne pour sa part que la plupart des gens adhérent spontanément à cette vision du système de justice (supra note 11 à la p 13). 
Or, comme le rappelle le professeur Georges De Leval, « comme tout service public, celui de la justice est au service du public. $\gg{ }^{153} \mathrm{Il}$ s'ensuit que le destinataire premier du service public de la justice, c'est le citoyen. Cette conception du citoyen ressort entre autres des propos de l'ancien ministre de la Justice du Québec Gil Rémillard, qui affirme ce qui suit : « (...) tout [le] système judiciaire doit exister en fonction, premièrement, du justiciable. L'institution est là pour servir le citoyen, et non pas le citoyen pour servir l'institution. $\gg{ }^{154}$ Elle est également visible à travers l'argumentaire du Comité d'action sur l'accès à la justice en matière civile et familiale, qui va jusqu'à poser le citoyen comme la raison d'être ${ }^{155}$ du système tout entier :

« Nous devons axer nos efforts sur les personnes qui utilisent le système. Cela doit comprendre toutes les personnes, en particulier les immigrants, les autochtones, les populations rurales et les autres groupes vulnérables. Les plaideurs, et en particulier ceux qui ne sont pas représentés par un avocat, ne sont pas, comme on les perçoit trop souvent, un inconvénient; ils sont la raison pour laquelle le système existe ${ }^{46} .{ }^{156}$

Cette affirmation est d'autant plus forte, lourde de sens, qu'elle fait du plaideur qui se présente seul en cour la raison d'être du système, et non pas simplement le justiciable représenté par avocat, intermédiaire par l'entremise duquel, traditionnellement, il s'exprime, agit en justice. C'est bien le citoyen lui-même qui est visé ici, directement. Partant, une réforme du système qui viserait à remettre le justiciable en son sein devrait voir à « détacher » celui-ci de la personne de son avocat, advenant qu'il en ait un ${ }^{157}$, détachement d'autant plus opportun que le meilleur intérêt de ce justiciable ne coïncide pas

153 De Leval, « citoyen », supra note 79 aux pp 45-46. Voir dans le même sens Commission on the Future of the Tennessee Judicial System, supra note 86 à la p 61.

154 Rémillard, supra note 57 à la p 22. Cette image du citoyen est également adoptée, entre autres, dans Bouchard, supra note 6 à la p 8; Lafond, L'accès à la justice civile, supra note 9 à la p 7, Buzzetti, supra note 6 et Boissavy et Clay, supra note 46 à la p 29, qui y voient au surplus une évidence : «Les justiciables sont les destinataires de la justice, elle est faite pour eux, et non contre eux. Et pourtant cette évidence n'est pas ce qui ressort des enquêtes d'évaluation et de satisfaction des citoyens face à la justice. » (nous soulignons).

155 Sur le citoyen comme raison d'être du système, voir aussi Kourlis et Olin, supra note 82 à la p 187; Boissavy et Clay, supra note 46 aux pp 20 et 23; Commission on the Future of the Tennessee Judicial System, supra note 86 à la p 7; Parker, supra note 11 à la p 5, et Margaret A Shone, Into the Future - Civil Justice Reform in Canada 1996 to 2006 and Beyond, décembre 2006, à la p 107 [Shone].

156 Comité d'action sur l'accès à la justice en matière civile et familiale, feuille de route, supra note 7 à la p 8 (nous soulignons). Mentionnons que la note 46 renvoit entre autres à Commission du droit de l'Ontario, «Pratiques exemplaires aux points d'entrée du système de justice familiale : besoins des utilisateurs et réponses des travailleurs du système de justice », Toronto, septembre 2009, à la p 11, en ligne, CDO : <http://www.lco- cdo.org/familylaw/Family\%20Law\%20Process\%20 Consultation\%20Paper\%20-\%20September\%20 2009_fr.pdf $>$ et Trevor Farrow et al, Répondre aux besoins des PNRA dans le système canadien de justice, Livre blanc préparé à l'intention de l'Association des administrateurs judiciaires du Canada, Toronto et Edmonton, 2012, aux pp 28 à 30 [Farrow et al]. Voir dans le même sens Emmanuelle Bernheim et Richard-Alexandre Laniel, « Un grain de sable dans l'engrenage du système juridique. Les justiciables non représentés : problèmes ou symptômes? », (2013) 31:1 Windsor YB Access Just 47, à la p 48 [Bernheim et Laniel], citant Lord Woolf, MR, Access to Justice - Interim Report to the Lord Chancellor on the civil justice system in England and Wales, 1996, para 2, tel qu'on l'a traduit à la page 34 de Farrow et al, cité ci-haut.

157 D'ailleurs, certains vont jusqu'à proposer une refonte du système en fonction des besoins des personnes non représentées plutôt que des justiciables représentés; voir par exemple Zorza, supra note 82 entre autres aux pp 11, 13, 18, 21 et 22, et 
forcément, on le sait, avec celui de son représentant ${ }^{158}$. La déclaration du Comité d'action est également importante en raison de la condamnation qu'elle contient. Elle rappelle en effet que les personnes non représentées sont trop souvent perçues comme un problème, voire un fardeau ${ }^{159}$ pesant sur les acteurs du système (avocats, juges et personnel judiciaire), alors qu'elles donnent à ce système tout son sens, fondent son existence même.

« Destinataires premiers » du service public de la justice, « raison d'être » du système judiciaire, les citoyens en seraient aussi, selon les tenants du recentrage, les véritables "propriétaires ${ }^{160}$. Il conviendrait de le rappeler, parce que la justice civile aurait tout d'un " domaine réservé »" ${ }^{161}$ aux seuls juristes, experts en droit. Dans les mots du professeur Alan Uzelac :

«The ecosphere of civil justice is all too often polluted by an eco-centric - or even egocentric - attitude, and the 'insider's' values often prevail over the values that serve the interests of users as 'one-shotters' and 'outsiders'. » ${ }^{162}$

Pierre Noreau dénonce également un certain repli de l'institution judiciaire sur elle-même, parlant d' « enfermement de la culture judiciaire ${ }^{163}$. Selon lui, cet enfermement s'est soldé par une justice qui, si elle devrait être « l'expression d'un consensus public », n'est plus aujourd'hui " que l'expression bureaucratique d'un système autopoïétique et satisfait, bref d'un champ d'action entièrement contrôlé

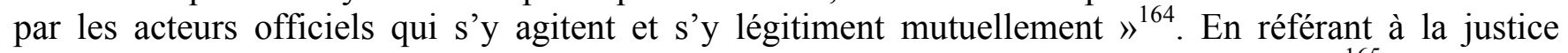
comme champ de pratique contrôlé par les juristes, Noreau évoque ici Pierre Bourdieu ${ }^{165}$, tandis que

Charles L Owen, Ronald W Staudt et Edward B Pedwell, Access to Justice. Meeting the Needs of Self-Represented

Litigants, Institute of Design and Chicago-Kent College of Law, Illinois Institute of Technology, 2001.

158 Voir par exemple Uzelac, « upside down », supra note 40 à la p 425.

159 Pour une condamnation de cette perception négative des justiciables non représentés, vus comme «éléments perturbateurs et perturbés », voir également Bernheim et Laniel, supra note 156, entre autres aux pp 53 et 54 . Pour les citoyens, plus généralement, vus comme « gêneurs » par les professionnels de la justice, voir encore Boissavy et Clay, supra note 46 à la p 23.

160 Voir par exemple Buzzetti, supra note 6; Boissavy et Clay, supra note 46 à la p 20; Chief Justice's Commission on the Future of the Courts, supra note 82 à la p 12 (« (...) courts belong to the public and not to its servants »), et Association du Barreau canadien, Justice pour tous, supra note 5 à la p 148 ( « Les Canadiens doivent percevoir que le système de justice, y compris les tribunaux, leur appartient $\gg)$.

161 L'expression est de Reid, supra note 136 à la p 51.

162 Uzelac, « Goals », supra note 78 à la p 27 (référence omise); voir également la page 28. Voir dans le même sens Canadian Bar Association, Task Force Report, supra note 9 à la p 17 : «The civil justice system has evolved over time as a peer-oriented system where operations are organized for the convenience of professional participants, that is, for lawyers, judges and court staff ».

163 Pierre Noreau, «Avenir de la justice : des problèmes anciens... aux solutions prochaines », dans Noreau, supra note 12 à la p 6 [Noreau, « des problèmes anciens »].

164 Noreau, «justice », supra note 6 à la p 41. Voir dans le même sens la page 51.

165 Il réfère d'ailleurs, à la page 51 de son article, à Pierre Bourdieu, « Les juristes gardiens de l'hypocrisie collective », dans François Chazel et Jacques Commaille, dir, Normes juridiques et régulation sociale, Paris, L.G.D.J., 1991, 95. Au sujet de la justice comme champ d'action selon Bourdieu, voir également Pierre Bourdieu, «La force du droit. Éléments pour une sociologie du champ juridique », (1986) 64 Actes de la recherche en sciences sociales 3, entre autres à la p 9 et $\mathrm{s}$. Voir également, toujours sur cette thèse de Bourdieu, Bernheim et Laniel, supra note 156 aux pp 50-51. 
l'idée de système autopoïétique fait écho, notamment, à Gunther Teubner ${ }^{166}$ ainsi qu'à Niklas Luhmann ${ }^{167}$.

Toujours selon Pierre Noreau, le phénomène de « cloisonnement $»^{168}$ de la justice civile s'explique en partie par l'institutionnalisation graduelle ${ }^{169}$ qu'elle a connue, un processus inévitable pour toute activité sociale et, au surplus, continu ${ }^{170}$. Cette institutionnalisation de la pratique et des structures judiciaires aurait été marquée, notamment, par ce que le sociologue appelle le procédé d'« auto-justification », qu'il décrit en ces termes :

« Toute institution, nous le savons, a pour fonction première de se maintenir, c'est-à-dire de se survivre à elle-même. La difficulté vient de ce que chacune d'entre elles crée sa propre rationalité. Elle justifie sa propre existence vis-à-vis de ses membres et vis-à-vis des autres institutions. Ces procédés d'auto-justification se rencontrent dans tous les champs d'activité organisés et les rendent partiellement étanches les uns aux autres, chacun cherchant à consacrer sa spécificité comme condition de son maintien et de son caractère indispensable pour les autres. C'est un des fondements de l'analyse systémique contemporaine. »

À ce procédé d'auto-justification s'ajoutent les «pratiques d'auto-production » que, selon lui, ne manque pas d'adopter chaque institution. Ces pratiques font en sorte que

« (...) plus une institution est ancienne, plus ses ressources servent au maintien de sa structure organisationnelle, jusqu'à ce que celle-ci devienne une justification en soi. Une bonne partie des problèmes que l'institution tente alors de résoudre est issue de son propre fonctionnement, de sa propre activité. Et plus l'institution est ancienne, plus elle est appelée à régler les problèmes qu'elle génère plutôt que les problèmes sociaux pour lesquels elle a été établie. ${ }^{171}$

Il en résulterait une institution judiciaire devenue, aujourd'hui, « sa propre cause, son propre produit et sa propre finalité ${ }^{172}$.

Pour se convaincre du fait que la justice civile semble aujourd'hui être l'apanage des juristes, il suffit de penser à la complexité inhérente au système judiciaire. Il est en effet de commune renommée que le langage, les règles de procédure, les rituels, en somme, la logique interne de ce système échappent aux

166 Auquel il réfère plus loin dans son article (à la p 52, n 37) : Gunther Teubner, Le droit, un système autopoïétique, coll. «Les Voies du droit », Paris, Presses universitaires de France, 1993. Sur les idées de Teubner à ce sujet, voir également Bernheim et Laniel, supra note 156 à la p 50.

167 Voir par exemple la définition de « système autopö̈́tique » contenue dans Niklas Luhmann, Essay on self-reference, New York, Columbia University Press, 1990, à la p 3, citée dans Bernheim et Laniel, supra note 156 à la p 50.

168 Noreau, « Avenir de l'institution judiciaire », supra note 6 à la p 60.

169 Sur la jusice comme institution sociale, et pour une définition de cette dernière notion, voir ibid à la p 53.

170 Ibid.

171 Ibid à la p 59.

172 Ibid. Y contribue également un autre procédé d'institutionnalisation, la « clôture des champs d'activités instituées », que l'auteur développe aux pages 59-60 et rapproche de l'auto-justification. 
non-initiés et que, en conséquence, il paraît tout entier pensé ou conçu pour les seuls spécialistes du droit $^{173}$. Pour le citoyen souhaitant ester en justice, cette complexité rend primordial le recours à l'avocat, sans l'aide duquel il s'avère hasardeux de "naviguer dans les méandres de la procédure civile ${ }^{174}$. Or, ce faisant, le justiciable se trouve relégué au rang de simple spectateur ${ }^{175}$, voire d'étranger ${ }^{176}$. Il devient « l'objet ${ }^{177}$ davantage que l'acteur d'un processus judiciaire qu'il a mis en branle, mais sur lequel il n'a pas de prise, de contrôle réel :

« (...) the present findings suggest a system where cases are no longer wholly "owned" by disputants, in some sense being co-opted from them, with decisions resulting in fundamental consequences for parties being taken out of their hands. As Felstiner et al. note, as a result of lawyers' monopoly on legal dispute processing coupled with the obscure nature of legal discourse and processes (...), "disputants' 'property' interests in disputes are appropriated by lawyers and the state". (...) [Using the law] increases their dependence on state institutions and comes at the price of domination by the state in the form of courts. To this, I would add "agents of the courts and court-linked processes." Thus, when plaintiffs move into the court system, they lose control over this power and become vulnerable to "the intervention of the rules and practices of the legal system and to the groups with the power to generate them." " ${ }^{178}$

173 Sur ce constat, voir notamment Comité d'action sur l'accès à la justice en matière civile et familiale, feuille de route, supra note 7 à la p 8; Association du Barreau canadien, Justice pour tous, supra note 5 p 66 et Canadian Bar Association, Task Force Report, supra note 9 à la p 17.

174 Pierre-Claude Lafond, Le recours collectif comme voie d'accès à la justice pour les consommateurs, Montréal, Thémis, 1996, à la p 79.

175 Voir notamment Boissavy et Clay, supra note 46 à la p 27 : « [Le justiciable] ne veut plus voir le palais de justice comme un théâtre dans lequel, après avoir remis entre les mains des acteurs de la vie judiciaire ses intérêts et sa liberté, il ne serait que simple spectateur de son propre destin. »

176 D'amour et al, « Nouveau Code de procédure civile », supra note 54 et Boissavy et Clay, supra note 46 à la p 108 (sur le système qui « maintient [le citoyen] à l'extérieur »). Voir également le constat en ce sens dressé dès $1978:$ « (...) Complicated procedures, detailed forms, (...) overbearing judges and lawyers make the litigant feel lost, a prisoner in an alien world. » (Mauro Cappelletti et Bryant G Garth, « Access to Justice: The Worldwide Movement to Make Rights Effective - A General Report », dans Mauro Cappelletti, dir, Access to Justice, vol. 1; Mauro Cappelletti et Bryant G Garth, dir, A World Survey, book 1, Alphenaandenrijn/Milan, Sithoff/Giuffrè, 1978,3, aux pp 16-17 [Cappelletti et Garth]).

177 Noreau, « justice », supra note 6 à la p 41. Voir également le passage suivant, tiré de Boissavy et Clay, supra note 46 à la p 23 : «"Corps présent”, c'est l'étrange expression qui désigne le justiciable assistant à son propre procès. Tout est résumé dans cette formule qui montre en quelle place l'institution judiciaire tient celui pour qui, pourtant, elle existe. Presque mortifère, la formule réduit le justiciable à l'état d'objet inerte dont la présence semble parfois gêner les professionnels de la justice qui préfèrent rester entre eux. »

178 Tamara Relis, Perceptions in Litigation and Mediation : Lawyers, Defendants, Plaintiffs and Gendered Parties, Cambridge; New York, Cambridge University Press, 2009, aux pp 238-239. Voir dans le même sens Laura Nader : «In Western societies the individual plaintiff has gradually been removed from litigation, (...) and has moved from a position of relative power, which gave him or her procedural means to dispute, to a relatively powerless role (...) » (Laura Nader, Harmony ideology: justice and control in a Zapotec mountain village, Stanford, Stanford University Press, 1990, à la p 160). 
Parce que la justice civile « concern[e] comme par essence l'intervention d'experts consacrés ${ }^{179}$, est « l'affaire des spécialistes ${ }^{180}$, des professionnels du droit ${ }^{181}$, il tombe sous le sens que sa réforme ait également été, historiquement, le domaine exclusif du milieu juridique, à l'exclusion des citoyens, profanes en droit $^{182}$. À ce propos, Hubert Reid fait remarquer de manière intéressante que si le tout premier Code de procédure civile, adopté en 1867, a été conçu par des juristes, ses réformes majeures subséquentes ont, elles aussi, été le fruit du travail exclusif de ceux-ci ${ }^{183}$. Il poursuit en notant que, lorsque le gouvernement juge opportun d'intervenir en matière de justice civile, il ne consulte généralement, à cette occasion, que les principaux intervenants du monde judiciaire ${ }^{184}$. L'auteur cite, à l'appui de son propos, le passage suivant d'une conférence prononcée en 1998 par le professeur Sylvio Normand, qui portait sur l'histoire de l'administration de la justice civile de 1840 à 1965 :

« Tout porte à croire que, au cours du processus de réforme du Code de procédure civile, seules sont retenues les modifications déjà largement acceptées par la communauté juridique québécoise. En somme, les acteurs en présence se voient reconnaître un droit de veto sur toute transformation substantielle de la procédure civile. Alors même que ce travail de réforme est conduit en plein cœur de la Révolution tranquille, la manière de fonctionner n'a guère changé par rapport à celle du siècle dernier. La réforme des institutions judiciaires est considérée comme une affaire interne au monde juridique, et ce, d'autant plus que le champ juridique a conservé une autonomie relative par rapport au pouvoir politique. $\gg{ }^{185}$

Dans ce contexte, l'Association du Barreau canadien, parmi d'autres, en appelle à une nécessaire appropriation du système de justice par le public :

« D'abord, nous devons écouter les points de vue du public et créer des tribunes inclusives pour le dialogue ainsi que des structures de reddition de comptes. Une véritable appropriation du système de justice par le public exigera toutefois plus que de meilleures consultations et un dialogue. Nous devons aussi transformer les structures de reddition de comptes pour inclure des représentants du public. Actuellement, les

179 Noreau, « justice », supra note 6 à la p 51.

180 Ibid. Voir également, dans le même sens, Daniel Jutras, « Culture et droit processuel : le cas du Québec », (2009) 54:2 McGill LJ 273, à la p 275.

181 Sur la professionnalisation et la spécialisation de la justice, voir par exemple Noreau, « Avenir de l'institution judiciaire », supra note 6 aux pp 54 et 61, et Noreau, « justice», supra note 6 aux pp 40-41 et 51.

182 Sur un tel constat, voir entre autres Lachapelle et al, « solutions », supra note 1; Lafond, L'accès à la justice civile, supra note 9 à la p 311; Observatoire du droit à la justice, supra note 54 à la p 9, et Noreau, « justice », supra note 6 à la p 51.

183 Tel que l'illustre la composition des comités de révision du Code. Voir Reid, supra note 136 à la p 51.

$184 \mathrm{Ibid}$. Voir dans le même sens la p 56. L'auteur donne l'exemple supplémentaire des colloques sur la justice civile, qui ne rassemblent généralement qu'une très grande majorité de juristes, juges, professeurs de droit et avocats au premier chef (ibid aux pp 51-52). Sur l'absence de consultation du public à l'occasion des réformes de la justice civile (ou le caractère exceptionnel de cette consultation populaire) constatée pour l'ensemble du Canada, voir notamment Canadian Bar Association, Task Force Report, supra note 9 à la p 17.

185 Sylvio Normand, « De la difficulté de rendre une justice rapide et peu coûteuse : une perspective historique (18401965) », (1999) 40 C de D 13, à la p 30. 
Canadiens croient que le système de justice appartient aux juges, aux avocats et au gouvernement. Il faut que cela change. Les Canadiens doivent percevoir que le système de justice, y compris les tribunaux, leur appartient. ${ }^{186}$

En résumé, les tenants de l'idée de recentrage ancrent leur argumentaire dans une vision de la justice civile qui constitue, par essence, un service public, service destiné d'abord et avant tout aux citoyens, qui donnent au système toute sa raison d'être et auxquels revient, en conséquence, la propriété d'un tel système. Nous verrons maintenant en quoi, toujours selon les défenseurs d'un recadrage de la justice civile autour du citoyen, cette vision du système de justice civile est aujourd'hui compromise en pratique.

\section{B. Le test de la réalité : le constat d'un décalage}

De nos jours, la justice civile se serait, dans les faits, éloignée de sa finalité première de service public; elle aurait perdu de vue son destinataire premier, le citoyen. C'est là l'avis, notamment, de l'ancien sous-ministre à la Justice Michel Bouchard, qui l'écrivait en 1999: "Tout semble indiquer qu'il y a un fossé entre la justice et ceux qu'elle devrait servir, et que ce fossé va en s'élargissant $»{ }^{187}$. Georges De Leval parle pour sa part d'une «fracture entre le citoyen et sa justice » ${ }^{188}$ et Pierre Noreau, d'une "distance $»{ }^{189}$. Indépendamment de la formule retenue pour l'exprimer, l'idée avancée par ces auteurs et d'autres ${ }^{190}$ est celle d'un citoyen qui n'est pas, en pratique, au centre du système de justice civile $^{191}$, alors qu'il devrait l'être.

Cette mise à distance est d'abord manifeste au regard des nombreuses barrières qui entravent la possibilité effective qu'ont les citoyens de s'adresser aux tribunaux. S'il est loin d'être nouveau, le problème de l'accès à la justice civile prend aujourd'hui, au Québec comme ailleurs, les proportions

186 Association du Barreau canadien, Justice pour tous, supra note 5 à la p 148 (nous soulignons). Voir dans le même sens la $\mathrm{p} 144$.

187 Bouchard, supra note 6 à la p 9.

188 De Leval, « citoyen », supra note 79 à la p 50.

189 Noreau, « justice », supra note 6 à la p 44. Il utilise également les expressions « mise à distance » et « distanciation » aux pp 40-41. Le phénomène de distanciation est également présenté dans Bernheim et Laniel, supra note 156 aux pp 50-51.

Voir aussi Commission on the Future of the Tennessee Judicial System, supra note 86 aux pp 11 et 13; Parker, supra note 11 à la p 29 et Boissavy et Clay, supra note 46 aux pp 23-24 (où les auteurs parlent d'une « distance assumée et insidieuse entre le justiciable et la justice qui éloigne celui-là de celle-ci. ») et 83 (où ils évoquent une « trop grande distorsion (...) entre la réalité et l'idéal »).

190 Voir entre autres Association du Barreau canadien, Justice pour tous, supra note 5 aux pp 6, 67, 70 et 144.

191 Voir notamment le constat en ce sens de l'Observatoire du droit à la justice : « Avec le temps, le justiciable a cessé d'être au centre du système judiciaire québécois ». Voir Oscar D’amours et al, « Plaidoyer pour un Livre blanc sur la justice Un souffle nouveau pour la justice québécoise », Le Devoir de Montréal (15 novembre 2010), en ligne :

$<$ http://www.ledevoir.com/societe/justice/310971/plaidoyer-pour-un-livre-blanc-sur-la-justice-un-souffle-nouveau-pourla-justice-quebecoise $>$ [D'amours et al, « Plaidoyer »]. Voir également le « lack of sufficient user-orientation » dénoncé dans Canadian Bar Association, Task Force Report, supra note 9 à la p 17 (où l'on pose l' « absence of a user-oriented or client-focused perspective » comme barrière systémique à l'accès à la justice en matière civile). Le même constat est posé en France par Boissavy et Clay, supra note 46 à la p 29 : « Malgré les efforts des magistrats, des avocats et du personnel judiciaire pour résoudre au mieux les litiges qui leur sont soumis, l'administration de la justice n'a pas encore placé le justiciable au centre de ses préoccupations. » 
d'une véritable crise ${ }^{192}$. Quels obstacles se dressent entre le citoyen et la justice civile ? Pensons d'abord aux coûts excessifs du système de justice (honoraires d'avocat, frais judiciaires, coûts d'expertise, déboursés, perte de salaire, etc.), et à ses délais, tout aussi importants. Pensons encore à la disponibilité territoriale et temporelle limitée des services judiciaires, ainsi qu'à la complexité et au formalisme procédural qui caractérisent la justice délivrée par les tribunaux. À ces obstacles objectifs, prohibitifs en soi, s'ajoutent des obstacles subjectifs ${ }^{193}$. Davantage méconnus bien que très importants ${ }^{194}$, ceux-ci concernent la connaissance et la perception qu'ont les citoyens des conditions d'accès aux tribunaux, connaissance et perception qui varient selon les traits individuels de ces citoyens (bagage socioculturel, âge, sexe, etc.) ${ }^{195}$. Parmi les barrières subjectives, citons notamment la complexité du langage juridique pour le citoyen, souvent profane en droit, l'absence ou l'insuffisance de l'information juridique dont il dispose, et les obstacles psychologiques qu'il rencontre (stress, caractère intimidant de l'enceinte judiciaire, etc.) ${ }^{196}$. Dans ce contexte, le citoyen se sent, à bon droit, tenu à l'écart d'un système judiciaire qui lui paraît - et, le plus souvent, lui est - fermé.

Plus fondamentalement (et peut-être plus problématiquement encore), la distance prise par la justice civile vis-à-vis de ses destinataires est également révélée par l'inadéquation du système et de ses procédures avec les besoins réels des citoyens, dont les attentes seraient continuellement frustrées. Dans les mots de Pierre-Claude Lafond : «Le système de justice, tel qu'on l'a connu jusqu'ici, souffre d'un problème d'adaptation aux besoins des citoyens pour lesquels il a été conçu ${ }^{197}$. Le même constat est posé à l'échelle du Canada par l'Association du Barreau canadien ${ }^{198}$ ainsi que par le Comité d'action sur l'accès à la justice en matière civile et familiale ${ }^{199}$, en plus de trouver écho ailleurs ${ }^{200}$. Partant, les citoyens ne se reconnaîtraient plus dans l'institution judiciaire ${ }^{201}$, celle-ci ne parvenant pas à les rejoindre ${ }^{202}$.

Il résulterait de cette justice civile aussi bien inaccessible qu'inadaptée une véritable crise de confiance. Selon l'Observatoire du droit à la justice, commentant la situation du Québec : « Les citoyens n’y croient plus. Toutes les études sociologiques réalisées au cours des 15 dernières années concluent

192 Voir notamment, pour ce constat posé au Québec, Lafond, L'accès à la justice civile, supra note 9 à la p 7.

193 D'après la dichotomie établie à l'origine par Monique Giard et Marcel Proulx, Pour comprendre l'appareil judiciaire québécois, Sillery, P.U.Q., 1985, à la p 244.

194 Au point de constituer, selon le Groupe de travail sur l'accessibilité à la justice s'étant penché sur la question en 1991, les plus importantes causes d'inaccessibilité. Voir Roderick A Macdonald, « Accessibilité pour qui ? Selon quelles conceptions de la justice? », (1992) 33 C de D 457, à la p 478.

195 Sur cette définition, voir entre autres Groupe de travail sur l'accessibilité à la justice, Rapport-synthèse - Jalons pour une plus grande accessibilité à la justice, Sainte-Foy, ministère de la Justice du Québec, 1991, à la p 2.

196 Voir notamment, pour un développement sur ces obstacles subjectifs et d'autres encore, Lafond, L'accès à la justice civile, supra note 9 aux pp 68-72.

197 Ibid à la p 7; voir également la page 297. Voir encore, dans le même sens, Bouchard, supra note 6 aux pp 8-9 et Noreau, «Avenir de l'institution judiciaire », supra note 6 aux pp 47 et 60 .

198 Association du Barreau canadien, Justice pour tous, supra note 5 aux pp 18 et 67.

199 Comité d'action sur l'accès à la justice en matière civile et familiale, feuille de route, supra note 7 aux pp iii et 1.

200 Voir, par exemple, le constat posé en France par l'ancien Premier Ministre Jean-Marc Ayrault (La Justice du $21^{e}$ siècle, les actes du débat national, supra note 98 à la p 13) et par Gaboriau, supra note 4.

201 Noreau, « justice », supra note 6 à la p 47. Voir aussi Boissavy et Clay, supra note 46 à la p 21.

202 Bouchard, supra note 6 à la p 9. 
dans le même sens : la confiance du public vis-à-vis des tribunaux passe rarement la barre des $50 \% »{ }^{203}$. Pire encore, le fait de bénéficier d'une expérience judiciaire accentuerait cette méfiance au lieu de la reléguer au rang de simple préjugé ${ }^{204}$. Le même constat est posé pour l'ensemble du Canada ${ }^{205}$, ainsi qu'en Belgique ${ }^{206}$ et en France ${ }^{207}$. Quant au Canada, il est intéressant de rapporter l'avis de l'Association du Barreau canadien selon lequel cette perte de confiance est directement fonction d'une absence « d'orientation-usager » en matière de justice civile : " the absence of a user-oriented or client-focused perspective is a leading cause of erosion of public confidence in legal institutions $\gg{ }^{208}$. Notons que cette crise de confiance trouve une illustration éloquente dans la désertion des tribunaux civils par le citoyen membre de la classe moyenne, un phénomène que certains qualifient de « décrochage judiciaire ${ }^{209}$.

En conséquence, les tribunaux accusant un « sérieux déficit de confiance » ${ }^{210}$, étant délaissés par ceux-là même auxquels ils sont destinés, ils seraient actuellement en proie à une profonde crise de légitimité $^{211}$. À ce propos, Pierre Noreau écrit : « (...) l'on mesure la légitimité d'une institution au besoin des citoyens d'y recourir dans la mise en forme de leurs rapports courants. La désuétude d'une institution se conclut en contrepartie de sa désertion graduelle par ceux qui devraient y recourir. ${ }^{212}$ Les tribunaux civils seraient-ils donc, aujourd'hui, désuets aux yeux des citoyens ? Chose certaine, la

203 Jacques Lachapelle et al, « Le monde judiciaire malade de sa justice », Le Devoir de Montréal (27 mars 2008), en ligne : $<$ http://www.ledevoir.com/non-classe/182276/le-monde-judiciaire-malade-de-sa-justice $>$. Notons toutefois que selon la plus récente étude menée par Pierre Noreau sur les attentes et les perceptions du citoyen vis-à-vis du système de justice, la confiance envers les tribunaux serait en hausse. Voir à ce propos Pierre Noreau, « Le citoyen et le système de justice québécois : analyse comparée des attentes et des perceptions de 1993 à 2014 », présentation donnée pour le compte du ministère de la Justice, 7 novembre 2014, à la p 13 (le texte de cette présentation nous a gracieusement été fourni par l'auteur).

204 Noreau, « des problèmes anciens », supra note 163 à la p 4. L'auteur ajoute que même l'opinion de ceux qui ont eu gain de cause en cour est négative (ibid). Ces constats ressortent également de l'enquête menée par le professeur en 2010 (Pierre Noreau, «La confiance du public : fondement de l'activité judiciaire », conférence prononcée lors du colloque pour l'accès à la justice organisé par la clinique juridique Juripop, présentée à Montréal, 4 mai 2012, aux p 11 et 15 (le texte de cette présentation nous a gracieusement été fourni par l'auteur)). Notons au surplus que même les professionnels du droit ont conscience de cette perte de confiance, jugeant qu'elle va jusqu'à menacer la pratique du droit (CIRANO, Enquête socio-économique auprès des membres du Barreau du Québec, 2008, en ligne, Barreau du Québec : $<$ www.barreau.qc.ca/barreau/donnees/index.html>).

205 Voir par exemple Association du Barreau canadien, Justice pour tous, supra note 5 aux pp 14-15, et Canadian Bar Association, Access to Justice Metrics, supra note 33 aux pp 24 et s.

206 Voir notamment De Leval, « citoyen », supra note 79 à la p 38 et à la p 31 : « Vue du côté du citoyen, la crise de la justice est avant tout une crise de confiance. ».

207 Voir entre autres La Justice du $21^{e}$ siècle, les actes du débat national, supra note 98, entre autres aux pp 16 et 26, et Gaboriau, supra note 4.

208 Canadian Bar Association, Task Force Report, supra note 9 à la p 17. Voir également la page 5.

209 C'est entre autres le cas de Lafond, L'accès à la justice civile, supra note 9 à la p 33. L'auteur rend compte du phénomène de désertion des tribunaux civils en chiffres (pp 33 à 45), avant d'aborder les causes de cette baisse de fréquentation (pp 45 à 47). Encore ici, cette désaffection ne serait pas propre au Québec, mais toucherait l'ensemble du Canada (Association du Barreau canadien, Justice pour tous, supra note 5 à la p 93).

210 Lafond, L'accès à la justice civile, supra note 9 à la $\mathrm{p} 7$.

211 Sur le constat d'une légitimité en panne posé au Québec, voir par exemple Noreau, " Avenir de l'institution judiciaire », supra note 6 aux pp 52-53 et 65-66; D'amours et al, « Plaidoyer », supra note 191 et Noreau, « justice », supra note 6 aux pp 33, 47 et 51. Pour cette même crise de légitimité constatée cette fois-ci en France, voir entre autres La Justice du $21^{e}$ siècle, les actes du débat national, supra note 98 notamment à la p 27 et Gaboriau, supra note 4.

212 Noreau, « justice», supra note 6 à la p 37. 
légitimité du système judiciaire est difficile à défendre dans le contexte actuel de désaffection populaire, si tant est que l'on adhère à l'idée, avancée par les tenants du recentrage, voulant que cette légitimité repose d'abord sur son utilisation effective par le public ${ }^{213}$ et sur la confiance que celui-ci lui accorde ${ }^{214}$.

Dans ce contexte, mettre le citoyen au cœur de l'institution judiciaire, ce serait « revenir aux finalités $»^{215}$ de cette institution, réaliser sa vocation première. Cela implique de combler l'écart entre la justice civile et le citoyen en cherchant à les rapprocher ${ }^{216}$, à créer les conditions nécessaires à l'avènement d'une véritable justice de proximité ${ }^{217}$. Ce rapprochement permet d'ancrer la justice civile dans le réel, en s'assurant qu'elle corresponde à la demande sociale, qu'elle soit le reflet de la population qu'elle dessert ${ }^{218}$; bref, qu'elle se révèle adaptée à la société et à ses évolutions récentes ${ }^{219}$. Le retour à la finalité première de la justice civile implique encore de combattre la désaffection populaire ${ }^{220}$ en tentant de regagner la confiance du citoyen ${ }^{221}$ et, partant, de restaurer une légitimité ${ }^{222}$ perdue, ou à tout le moins menacée. Ce faisant, c'est l'idéal démocratique lui-même qui s'en trouve consolidé2 23 .

213 Voir par exemple Bouchard, supra note 6 à la p 8 : « Pourtant, comment pourrions-nous justifier toute notre organisation de la justice si aucun citoyen ne faisait appel à ces services?».

214 Voir par exemple Noreau, « Avenir de l'institution judiciaire », supra note 6 à la p 66.

215 Ibid. Voir également Lachapelle et al, « solutions », supra note 1 et Bernheim et Laniel, supra note 156 à la p 66.

216 Boissavy et Clay, supra note 46 à la p 95. Voir aussi la page 94 et les pp 117 et $\mathrm{s}$.

217 Notons que l'idée de proximité est très présente dans les sources françaises consultées. Voir à ce propos, notamment, Boissavy et Clay, supra note 46 aux pp 117 et s; Commission sur la répartition des contentieux, supra note 6 entre autres aux pp 34-35 et 41-42; La justice du 21 siècle, les scénarios de réforme, supra note 100 aux pp 3 et 6-7; La Justice du $21^{e}$ siècle, les actes du débat national, supra note 98 entre autres aux pp 16, 19, 26 (où la proximité est posée comme demande citoyenne) et 29 (où la proximité, lorsqu'elle est comprise intellectuellement (et non pas géographiquement) est définie comme «l'attention à toutes les particularités » et est liée à un individualisme entendu positivement, exprimant « le fait que chacun souhaite être reconnu dans sa spécificité, dans sa dignité, qu'il soit respecté. » (p 30)); voir également le développement de la notion de justice de proximité aux pp 75 et s; voir aussi Gaboriau, supra note 4.

218 Comité d'action sur l'accès à la justice en matière civile et familiale, feuille de route, supra note 7 à la p $15:$ : Le continuum de la justice doit refléter la population qu'il dessert ».

219 Voir par exemple Commission sur la répartition des contentieux, supra note 6 aux pp 29 et 35-36; Noreau, « justice », supra note 6 aux pp 51-52; Choquette, supra note 43; Uzelac, « Goals », supra note 78 à la p 10; Uzelac, « upside down », supra note 40 aux pp 414-415; Gaboriau, supra note 4 et Noreau, « Avenir de 1'institution judiciaire », supra note 6 aux pp 41 et 54 .

220 Voir entre autres Comité de révision de la procédure civile, supra note 6 à la p 32; Association du Barreau canadien, Justice pour tous, supra note 5 à la p 93, et Bouchard, supra note 6 à la p 8.

221 Parce qu' « il n'y a pas de justice et il n'y a pas de système judiciaire sans la confiance du public » (Noreau, « Avenir de l'institution judiciaire », supra note 6 à la p 65). Voir également, sur la nécessité de rétablir cette confiance, Comité de révision de la procédure civile, supra note 6 à la p 32; Association du Barreau canadien, Justice pour tous, supra note 5 aux pp 66 et 93, et Shone, supra note 155 à la p 108. Pour la même idée avancée à l'étranger, voir encore Chief Justice's Commission on the Future of the Courts, supra note 82 sous « vision » (page non numérotée); Zorza, supra note 82 à la p 7; Commission on the Future of the Tennessee Judicial System, supra note 86 aux pp 11 et 33; Parker, supra note 11 aux pp v, 15, 26 et 28-29; De Leval, « citoyen », supra note 79 à la p 40; La Justice du $21^{e}$ siècle, les actes du débat national, supra note 98 aux pp 16 et 26 (où la confiance est posée comme « institution invisible », empruntant l'expression du prix Nobel d'économie Kenneth Arrow), et Gaboriau, supra note 4.

222 La Justice du $21^{e}$ siècle, les actes du débat national, supra note 98 aux pp 16, 20, 26 (où la légitimité est posée comme « institution invisible », empruntant l'expression du prix Nobel d'économie Kenneth Arrow) et 27-28 (où l'on distingue la légitimité procédurale, la légimité substantielle et la légitimité d'exercice); Lachapelle et al, " solutions », supra note 1; Noreau, «Avenir de l'institution judiciaire », supra note 6 aux pp 52-53 et 69; Association du Barreau canadien, 
Qui plus est, recentrer la justice civile autour de la personne du citoyen, c'est aussi faire en sorte de lui rendre son système de justice civile ${ }^{224}$. C'est plaider, comme le fait l'Association du Barreau canadien, pour une appropriation du système de justice par le public, système trop longtemps confisqué, à tort, par les juristes, alors même que sa propriété véritable reviendrait de droit aux citoyens ${ }^{225}$. C'est encore, comme l'affirme le Comité d'action sur l'accès à la justice en matière civile et familiale, s'assurer que le système fonctionne réellement pour ceux qui l'utilisent, et non pour les membres de la profession juridique ${ }^{226}$.

Pour ce faire, les tenants du recentrage plaident pour ce que nous appellerons un déplacement du regard. Ils invitent en effet les architectes d'une réforme future de la justice civile à se détourner des besoins et impératifs du système et de ses acteurs (avocats, juges et personnel judiciaire) - lesquels ont, jusqu'ici, dominé, au point d'occulter tout le reste - pour considérer plutôt ceux du citoyen, à titre de membre de la société civile aussi bien que d'usager éventuel du système ${ }^{227}$. Précisons qu'il ne s'agit pas d'évacuer complètement les juristes et leurs intérêts propres de l'équation ${ }^{228}$. En d'autres termes, le citoyen ne devient pas l'unique joueur à prendre en considération. L'idée est plutôt, ici, de lui donner la priorité, laquelle allait, jusque-là, aux experts en droit. Rappelons à ce propos la formulation de l'idée de recentrage que met de l'avant le Comité d'action sur l'accès à la justice en matière civile et familiale : «donner la priorité au public » ${ }^{229}$. Cette idée de priorité ressort également de la formule employée par

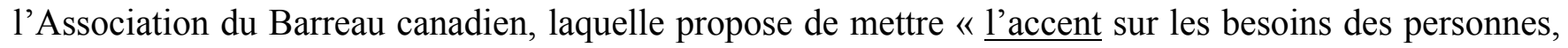

Justice pour tous, supra note 5 aux pp 14-15; Noreau, « justice », supra note 6 à la p 51; Gaboriau, supra note 4; Shone, supra note 155 à la $\mathrm{p} 108$, et Parker, supra note 11 à la $\mathrm{p} 15$.

223 Voir notamment La Justice du $21^{e}$ siècle, les actes du débat national, supra note 98, à la p 26; Commission sur la répartition des contentieux, supra note 6 à la p 29; Lachapelle et al, " solutions », supra note 1; Choquette, supra note 43; Association du Barreau canadien, Justice pour tous, supra note 5 aux pp 54, 55 et 93; Boissavy et Clay, supra note 46 aux pp 10, 14, 20 et 227; British Columbia Ministry of Justice, White Paper, supra note 9 à la p 4; Zorza, supra note 82 à la p 7; Shone, supra note 155 à la p 108, et Parker, supra note 11 aux pp 28-29.

224 Buzzetti, supra note 6.

225 Voir ci-dessus, page 34.

226 Comité d'action sur l'accès à la justice en matière civile et familiale, feuille de route, supra note 7 à la p 8 . Voir dans le même sens Cappelletti et Garth, supra note 176 à la p 5 : «No aspect of our modern legal systems is immune from criticism. Increasingly it is asked how, at what price, and for whose benefit, do these systems really work; (...) » (nous soulignons).

227 Voir notamment Comité d'action sur l'accès à la justice en matière civile et familiale, feuille de route, supra note 7 aux pp 2 et 8; Association du Barreau canadien, Justice pour tous, supra note 5, aux pp 65, 66 et 68 et Action committee on access to justice in civil and family matters, Prevention, triage and referral working group, supra note 12 aux pp i et 3. Voir également Lachapelle et al, « solutions », supra note 1, sous « rompre avec l'enfermement ».

228 Voir, par exemple, Comité d'action sur l'accès à la justice en matière civile et familiale, feuille de route, supra note 7 à la p 2 : «Bien entendu, [1]e système officiel est important. Mais une vision plus large, et qui soit basée sur les utilisateurs, d'un système de justice en matière civile et familiale accessible est nécessaire. » Voir dans le même sens Action committee on access to justice in civil and family matters, Prevention, triage and referral working group, supra note 12 à la $\mathrm{p} 3$.

229 Comité d'action sur l'accès à la justice en matière civile et familiale, feuille de route, supra note 7 à la p 8 (nous soulignons). L'idée de priorité ressort également lorsque le Comité parle de « modifier la cible principale » des efforts en matière d'accès à la justice, d' « axer » ceux-ci sur les usagers (ibid). Voir encore, sur l'idée de priorité, Action committee on access to justice in civil and family matters, Prevention, triage and referral working group, supra note 12 aux pp i, 3 et 35 et Chief Justice's Commission on the Future of the Courts, supra note 82 à la p 12. 
et non ceux des professionnels de la justice et des institutions ${ }^{230}$. C'est donc dire que l'idée de recentrage implique une certaine hiérarchisation des intérêts des divers acteurs en cause, hiérarchisation qui devra s'opérer en faveur du citoyen.

Au plan méthodologique, le déplacement du regard prôné par les tenants d'un recentrage implique la définition d'un nouveau point de départ, celui des attentes et des besoins du citoyen en matière de justice civile, attentes et besoins dont l'identification est posée comme préalable nécessaire à toute entreprise de réforme $^{231}$. À son tour, cette étape préalable suppose de se mettre à la place de ce citoyen ${ }^{232}$, d'adopter son point de vue, sa perspective ${ }^{233}$. Bref, on invite en quelque sorte les artisans d'une réforme éventuelle à se faire citoyens ${ }^{234}$. En cela, c'est à une véritable " révolution des esprits ${ }^{235}$ juridiques que nous convient les tenants du recentrage.

Évidemment, cette " révolution " ne se fera pas sans heurts, à supposer même qu'elle se fasse. Plusieurs obstacles viennent spontanément à l'esprit lorsque l'on songe à la mise en pratique d'une idée aussi ambitieuse que celle de placer le citoyen au cœur du système, obstacles que le présent article ne nous aura pas permis d'aborder. Parmi ces difficultés à prévoir et à surmonter, on peut faire mention, par

230 Association du Barreau canadien, Justice pour tous, supra note 5 à la p 65 (nous soulignons). Voir, dans le même sens, British Columbia Ministry of Justice, White Paper, supra note 9 aux pp 3 et 7.

231 Sur l'identification des besoins juridiques comme préalable à toute réforme en matière civile, voir notamment la méthodologie employée par le HiiL, décrite dans Barendrecht et al, supra note 12 aux pp 3, 15 et 22. L'idée de poser les besoins et les attentes du citoyen comme point de départ est également défendue dans Action committee on access to justice in civil and family matters, Prevention, triage and referral working group, supra note 12 aux pp i, 3 et 35 ; Roberge, «perspectives », supra note 28 aux pp 13 et 27; Comité d'action sur l'accès à la justice en matière civile et familiale, feuille de route, supra note 7 à la p 2, et Association du Barreau canadien, Justice pour tous, supra note 5 à la p 148. Précède l'identification des attentes et des besoins, celle des usagers eux-mêmes; on ne peut recentrer le système autour de ceux-ci si on ne s'intéresse pas à qui ils sont, si on ne s'attarde pas à les comprendre. Voir Parker, supra note 11 aux pp 12-13, et Boyle, supra note 39.

232 Commission sur la répartition des contentieux, supra note 6 à la p 183. Voir aussi Boyle, supra note 39.

233 Voir par exemple le jalon de réforme suivant, posé par l'Association du Barreau canadien : « La réforme de la justice tient compte de la perspective du public, qui oriente l'élaboration des politiques et des processus, l'innovation et la réforme dans le système de justice. » (Association du Barreau canadien, Justice pour tous, supra note 5 à la p 148). Voir, dans le même sens, Barendrecht et al, supra note 12 aux pp 3, 15 et 22; Comité d'action sur l'accès à la justice en matière civile et familiale, feuille de route, supra note 7 aux pp 2 et 17; Action committee on access to justice in civil and family matters, Prevention, triage and referral working group, supra note 12 aux pp i et 3 et Roberge, « perspectives », supra note 28 aux pp 13 et 27.

234 C'est ce qu'a tenté de faire la Commission on the Future of the Tennessee Judicial System dans son rapport; voir supra note 86 à la p $66:$ « (...) we tried throughout to approach the subject from our perspective as citizens. 》 (nous soulignons).

235 De Leval, « citoyen », supra note 79 à la p 50 et Boissavy et Clay, supra note 46 à la p 29. Sur l'inévitabilité d'un important changement de culture pour que se concrétise, en pratique, l'idée de recentrage, voir également Canadian Bar Association, Task Force Report, supra note 9 à la p 56; Comité d'action sur l'accès à la justice en matière civile et familiale, feuille de route, supra note 7 aux pp iv et 8 (posant la priorité donnée au public comme principe directeur d'une changement de culture plus large, rappelons-le; voir ci-dessus, page 5); Lachapelle et al, " solutions », supra note 1; D'amour et al, « Nouveau Code de procédure civile », supra note 54; Comité d'action sur l'accès à la justice en matière civile et familiale, Compte rendu du Colloque, supra note 12 à la p 13; British Columbia Ministry of Justice, White Paper, supra note 9 à la p 7, et Parker, supra note 11 à la p 22. 
exemple, de la résistance que l'idée rencontrera certainement chez les juristes ${ }^{236}$. Après tout, voilà des années que l'on plaide pour un nécessaire «changement de culture » en matière de procédure civile, sans que celui-ci ne se soit encore véritablement concrétisé ${ }^{237}$. Partant, il est permis de se demander ce qui pousse à être plus optimiste dans le cas d'une évolution des mentalités ne s'appliquant plus simplement aux pratiques ayant cours en matière procédurale, mais bien à la conception même du système judiciaire.

Plus fondamentalement encore, c'est toute la question de savoir si l'idée de «placer le citoyen au cœur du système » peut, oui ou non, trouver un véritable sens pratique que le présent article n'aura pas explorée. En effet, si acquiescer dans l'absolu à l'idée de recentrage est une chose, saisir pleinement ce que cette prise de position implique au plan pratique en est une autre. Au-delà de la nécessaire rencontre des « attentes et besoins » des citoyens - une formule tellement répandue qu'elle en est presque devenue galvaudée - qu'est-ce que signifie, concrètement, l'idée de mettre ces citoyens au centre du système ? Cette idée n'est-elle, à bien y regarder, qu'un discours vide, de belles paroles dénuées de toute portée réelle ? N'y a-t-il là qu'un idéal par essence inatteignable, ou encore qu'un message politique semblable à une promesse électorale qui restera sans suite ? Ou cette idée est-elle, au contraire, suffisamment précise, incarnée, pour être posée en objectif véritable et réalisable ? Des initiatives comme le projet de loi 661 en France nous donnent l'espoir d'une mise en pratique possible, faisable, de l'idée de recentrage. L'avenir nous dira si d'autres juridictions suivront le pas et feront en sorte de concrétiser cette idée fondamentale sur le plan de la démocratie.

236 En effet, si le discours de recentrage émane en partie, on l'a vu, des acteurs judiciaires eux-mêmes, c'est probablement également au sein de ceux-ci que l'on compte ses principaux opposants. Dit autrement, l'idée de recentrer le système autour de l'usager dérange surtout, à notre avis, ceux qui sont habitués au système dans sa forme actuelle.

237 Quoi qu'en disent ceux qui sont d'avis que le nouveau Code de procédure civile réalise, au Québec, ce changement de culture. 